\title{
Pourquoi respectons-nous la soft law ?
}

Le rôle des émotions et des techniques de manipulation

\section{Alexandre Flückiger}

\section{OpenEdition}

\section{Journals}

Édition électronique

URL : http://journals.openedition.org/ress/68

DOI : $10.4000 /$ ress.68

ISSN : 1663-4446

Éditeur

Librairie Droz

\section{Édition imprimée}

Date de publication : 1 mai 2009

Pagination : 73-103

ISBN : 978-2-600-01303-1

ISSN : 0048-8046

\section{Référence électronique}

Alexandre Flückiger, "Pourquoi respectons-nous la soft law ?», Revue européenne des sciences sociales [En ligne], XLVII-144 | 2009, mis en ligne le 01 mai 2012, consulté le 19 avril 2019. URL : http:// journals.openedition.org/ress/68; DOI : 10.4000/ress.68 


\section{Alexandre FLÜCKIGER}

\section{POURQUOI RESPECTONS-NOUS LA SOFT LAW? Le rôle des émotions et des techniques de manipulation}

\section{INTRODUCTION ${ }^{1}$}

\subsection{Le respect du droit}

«D'où vient cette singulière habitude de respecter les lois, auxquelles seuls quelques «anormaux» n'obéissent que contraints?» ${ }^{2}$. Des sanctions coercitives qui y sont rattachées? De la peur qu'elles provoquent? Des menaces qu'elles représentent? De la culpabilité et de la honte qu'elles engendrent? En bref: des émotions qu'elles peuvent faire naître?

En partie certainement, mais pas uniquement. L'autorité de la loi ne provient pas de la seule coercition. D'une part, la masse des lois, et le volume de jurisprudence associée, ont pour conséquence que la plupart des règles de droit sont certainement respectées sans conscience dans le public de leur existence précise. D'autre part, le droit ne se définit aujourd'hui plus par le seul critère de la sanction strictement définie ${ }^{3}$. Les explications vont dès lors foisonner et différer selon les points de vue analysés: les sociologues évoqueront les normes sociales ${ }^{4}$, les éthiciens le sens des valeurs, les théologiens la morale, les économistes l'efficience ${ }^{5}$, les psychiatres le surmoi, les neuroscientifiques l'activation de

1 Ce texte présenté dans le séminaire organisé par la Fondation des Treilles à Tourtour sur le thème «Rationalité et émotions » (juillet 2008) s'inscrit dans le cadre d'une recherche financée par le Fonds national suisse de la recherche scientifique consacrée au «rôle des émotions dans les processus de régulation juridique et sociale» (programme national de recherche en sciences affectives) dont il constituera une partie du rapport final.

2 Gaudemet, J., cit. in Papaux, Alain, Essai philosophique sur la qualification juridique: de la subsomption à l'abduction: l'exemple du droit international privé, Bruxelles [etc.] 2003, p. 215.

3 Béchillon, Denys de, Qu'est-ce qu'une règle de droit?, Paris 1997, p. 61; Morand, CharlesAlbert, Le droit néo-moderne des politiques publiques, Paris 1999, p. 173; Timsit, Gérard, Archipel de la norme, Paris 1997, p. 176ss; Ost, François/ Kerchove, Michel van de, De la pyramide au réseau? Pour une théorie dialectique du droit, Bruxelles, 2002, p. 222ss; Millard, Eric, Théorie générale du droit, Paris 2006, p. 35 (qui compte la «sanction» sociale comme raison donnant son effectivité aux règles de droit). Voir également Papaux, Alain, Introduction à la philosophie du «droit en situation»: de la codification légaliste au droit prudentiel, Bruxelles [etc.] 2006, p. 24ss, qui utilise la définition classique du droit coercitivement sanctionné comme «un échafaudage qu'il faut savoir abandonner une fois l'édifice (de la connaissance du droit) érigé» (p. 25).

4 Le thème a été repris par les juristes (voir Posner notamment [réf. cit. ci-dessous note 46]).

5 Voir en particulier les travaux en law and economics (réf. cit. ci-dessous note 29). 
zones cérébrales spécifiques à l'obéissance aux normes ${ }^{6}$, les psychologues les émotions ${ }^{7}$, etc.

\subsection{Le respect de la soft law}

Si le respect du droit (dur) ne se laisse pas si simplement expliquer, celui du droit souple (soft law) l'est encore moins. N'est-il en effet pas étonnant, pour un esprit juridique tout au moins, qu'un certain nombre d'actes dénué de caractère obligatoire $^{8}$, telles que des recommandations, des déclarations, des activités d'information et de persuasion, des gentlemen's agreement, des chartes ou d'autres actes incitatifs puissent forcer le respect? Les juristes internationalistes ont depuis une trentaine d'année thématisé juridiquement l'émergence d'une soft law, s'éloignant d'une conception du droit monolithique et hiérarchique pour reconnaître l'existence, en marge, d'un droit plus souple dont la pertinence juridique est difficilement contestable ${ }^{9}$. Alors que ce phénomène en droit interne était déjà connu bien antérieurement $^{10}$, il n'a été thématisé que progressivement et n'a pas encore rallié l'entier de la doctrine derrière son étendard. La difficulté conceptuelle majeure provient du fait qu'admettre la soft law comme source dans la grande rivière du droit, fût-elle d'un petit affluent, implique d'admettre l'hypothèse de l'existence d'une normativité graduelle, alors que les défenseurs de l'existence d'un «seuil normatif» sont encore nombreux ${ }^{11}$. Admettre une gradation du phénomène normatif conduit à admettre non seulement l'existence de degrés dans les actes non obligatoires, mais également dans les actes de hard law chaque fois que l'on est en présence de concepts juridiques indéterminés ou de normes juridiques non justiciables ${ }^{12}$.

${ }^{6}$ Spitzer, M./ Fischbacher, U./ Herrnberger, B./ Grön, G./ Fehr, E., «The neural signature of social norm compliance», Neuron 2007, 56, p. 185ss. En neuro-marketing (lien entre des actes d'achat et des zones cérébrales), voir Lee, N./ Broderick, A.J./ Chamberlain, L., «What is «neuromarketing»? A discussion and agenda for future research », International journal of psychophysiology 2007, 63, p. 199ss.

7 Voir ci-dessous ch. 2.2.

${ }^{8}$ Le caractère obligatoire allié à celui de la justiciabilité peut être considéré comme définissant plus sûrement la notion de droit que celui de la sanction (Ost/Kerchove 2002 [note 3], p. 302ss; Feuz, Roland, Materielle Gesetzesbegriffe: Inhalt und Tragweite: dargestellt insbesondere anhand von Art. 164 Abs. 1 derneuen Bundesverfassung, Berne 2002,p. 65ss; Morand 1999 [note 3], p. 173; Amselek, Paul, Méthode phénoménologique et théorie du droit, Paris 1964, p. 264ss. Voir ég. note 3 ci-dessus. Sur la reconnaissance en droit suisse de la pertinence juridique de tels actes étatiques non obligatoires, voir notamment Richli, Paul, «Öffentlich-rechtliche Probleme bei der Erfüllung von Staatsaufgaben mit Informationsmitteln », Revue de droit suisse I 1990, p. 151 ss ; Müller, Markus/ Müller-Graf, Thomas, «Staatliche Empfehlungen: Gedanken zu Rechtscharakter und Grundrechtsrelevanz», Revue de droit suisse II 1995, p. 357 ss ; Mahon, Pascal, «L'information par les autorités», Revue de droit suisse II 1999, p. 199 ss; Tschannen, Pierre, «Amtliche Warnungen und Empfehlungen», Revue de droit suisse II 1999, p. 353 ss.

${ }^{9}$ Shelton, Dinah (éd.), Commitment and Compliance: The Role of Non-Binding Norms in the International Legal System, Oxford 2000.

${ }^{10}$ Les internationalistes se sont référés aux règles étatiques internes non obligatoires pour justifier la prise en considération juridique de la soft law (réf. in Flückiger 2004, p. 224ss).

${ }^{11}$ Sur le débat, voir Flückiger, Alexandre, «Régulation, dérégulation, autorégulation: l'émergence des actes étatiques non obligatoires », Revue de droit suisse II, 2004, p. 235s ; Culot, Henri, «Soft law et droit de l'OMC», Revue internationale de droit économique, 2005, p. 251ss, 284.

${ }^{12}$ Hard law «diminuée» que certains internationalistes comprennent sous l'étiquette de soft law du point de vue du contenu (soft law matérielle: voir Flückiger 2004 [note 11], p. 226s). 
Abandonnant provisoirement ces considérations dogmatiques pour le terrain de l'observation empirique, on constate que les règles de soft law peuvent en pratique être respectées indépendamment de leur caractère juridique ou non. À des degrés divers certes, mais sans différer fondamentalement sur cet aspect des actes obligatoires dont le degré d'observation est éminemment variable: «In some instances, compliance with non-binding norms and instruments is extremely good and probably would not have been better if the norms were contained in a binding text.» ${ }^{13}$ La Déclaration commune des Ministres européens de l'éducation réunis à Bologne le 19 juin 1999 (Déclaration de Bologne), acte non obligatoire, est un exemple de la puissance potentielle des effets de la soft law. Ce texte a été suivi et mis en œuvre bien plus efficacement et rapidement dans le paysage universitaire ${ }^{14}$ que la règle frappant de contravention le piéton marchant sur une piste cyclable alors qu'il dispose d'un trottoir (art. 40 al. 2 OCR $^{15}$, annexe I OAO ${ }^{16}$ ). C'est le constat d'un phénomène singulier pour un juriste: les règles de droit, bien qu'obligatoires, sont parfois moins bien respectées que des règles de soft law même s'il est indéniable que les règles de hard law sont souvent plus efficaces que celles de soft law prises isolément ${ }^{17}$, et que la soft law ne s'avère de loin pas toujours aussi efficace $^{18}$. L'effet le plus marqué étant certainement une combinaison entre les règles de droit, leur contrôle et les instruments de soft law: en matière de délinquance routière, les campagnes d'information permettent ainsi d'augmenter l'effet des programmes policiers ${ }^{19}$. Ni la variable du caractère obligatoire, ni celle de la sanction ne sont donc à elles seules déterminantes dans l'analyse du respect des règles.

Cette observation rappelle que le registre normatif, contrairement à celui des faits, laisse à l'individu la liberté de se déterminer selon son libre arbitre, donc de désobéir ${ }^{20}$. Les actes matériels à caractère contraignant de fait, tels les obstacles à la circulation (seuils de ralentissement du trafic), les instruments techniques de limitation de l'usage d'Internet (code based regulation) ou des voitures (blocage

13 Shelton, Dinah, «Soft Law», Handbook of International Law, Routledge Press, 2008, ch. 4.

${ }^{14}$ Sur la puissance de l'effet de cette déclaration en droit suisse, voir Ehrenzeller, Bernhard/ Reisner Annegret, ,Die Konstitutionalisierung von «Bologna»: Sonderfall oder Modell der Weiterentwicklung des kooperativen Föderalismus?«, Revue de droit suisse 2008 II, p. 229ss.

${ }^{15}$ Recueil systématique (ci-après: RS) 741.11 (www.admin.ch/ch/f/rs).

${ }^{16}$ RS 741.031.

17 Voir par exemple les études montrant que l'existence d'une obligation de porter la ceinture de sécurité entraîne une modification du comportement beaucoup plus importante qu'une simple recommandation (Killias, Martin, Précis de criminologie, $2^{\mathrm{e}}$ éd., Berne 2001, p. 453ss).

${ }^{18}$ Voir par exemple le gentlemen's agreement passé en février 2002 entre le Département de l'environnement, des transports, de l'énergie et de la communication (DETEC) et l'association des importateurs suisses d'automobiles (auto-suisse) qui définissait un objectif de réduction de la consommation des voitures de tourisme neuves de $3 \%$ par an en moyenne et qui devait permettre d'abaisser leur consommation à 6,4 1 aux $100 \mathrm{~km}$ d'ici 2008. Cet objectif ne pourra être atteint si bien que la Confédération prévoit d'introduire des «mesures plus énergiques» (DETEC, Communiqué de presse du 15 mai 2008); mesures dont l'efficacité restera bien sûr à prouver.

${ }^{19}$ Blais, Etienne, «L'effet des lois et des contrôles pénaux sur la délinquance et les accidents de la circulation », in Strassenverkehr, Auto und Kriminalität, Berne 2008, p. 155ss.

${ }^{20}$ Ost/Kerchove 2002 [note 3], p. 367. 
du démarrage en l'absence de ceinture de sécurité attachée) ne sauraient donc être comptés au rang de la soft law, n'étant ni law ni $s o f t^{21}$. La confusion provient de l'amalgame courant entre la notion de nécessité et celle d'obligation ${ }^{22}$ : si le véhicule est construit pour ne pas démarrer en l'absence de ceinture de sécurité, l'automobiliste devra nécessairement l'attacher alors que si seule une règle de droit lui impose le port de la ceinture, l'automobiliste devra obligatoirement - seulement - la porter, conservant toujours la possibilité de violer la règle de droit en assumant le risque des conséquences juridiques qui en découlent.

\subsection{La nécessité de légitimer l'action non normative de l'Etat}

Comme une règle non obligatoire peut parfois suffire à modifier le comportement des individus, l'Etat a pris l'habitude d'y recourir en plus - ou à la place d'actes juridiques classiques. Cela lui permet d'éviter de suivre les lourdes procédures juridiques applicables aux règles de droit et, de plus, de renforcer son emprise. Le phénomène n'est pas nouveau. On en trouve par exemple trace dans la notion de gouvernementalité de Michel Foucault ${ }^{23}$. L'originalité réside toutefois dans la conscience qu'ont désormais les juristes de la force de ces instruments «faibles »; le système juridique s'étant un peu commodément retranché derrière le prétexte du caractère non juridique pour éviter de se prononcer sur la légalité ou la constitutionnalité de tels instruments. L'enjeu consiste donc à convaincre l'Etat de développer des mécanismes de légitimation adaptés, comme le droit constitutionnel, par le passé, l'a fait en démocratisant la loi, en imposant à celle-ci des exigences de précision et en la soumettant au contrôle de constitutionnalité ${ }^{24}$, ou comme le droit administratif en exigeant des garanties de procédure pour l'adoption des décisions administratives ou de certains plans.

C'est dans ce but que l'on explorera ici les mécanismes d'influence de la soft law. Nous les détaillerons sous l'angle psychologique. On distinguera le rôle des émotions dans les techniques de persuasion (ch. 2) et dans celles de manipulation permettant elles d'influencer le comportement des individus à leur insu (ch. 3).

\section{LE RÔLE DES ÉMOTIONS DANS LES TECHNIQUES DE PERSUASION}

La notion d'émotion ne se laisse pas aisément définir. Certes nombreuses sont encore aujourd'hui les références faites aux six émotions de bases de Paul Ekman que sont la colère, la peur, le dégoût, la joie, la tristesse et la surprise ${ }^{25}$. La

${ }^{21}$ Voir Flückiger 2004 [note 11], p. 183ss.

${ }^{22}$ Sur cette confusion, voir Lalande, André, Vocabulaire technique et critique de la philosophie,

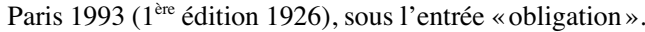

${ }^{23}$ Voir Flückiger 2004 [note 11], p. 170ss.

${ }^{24}$ Sur ce dernier point, la situation est perfectible en Suisse s'agissant des lois fédérales, soumises à un contrôle limité (voir art. $190 \mathrm{Cst}$.).

${ }^{25}$ Ekman, Paul, «An Argument for Basic Emotions », Cognition and Emotion, 1992, 6, 169ss; Ekman, Paul, Emotions in the Human Face, Cambridge 1982. 
recherche dans le domaine n'a fait cependant qu'en complexifier le concept en les distinguant d'autres affects tels que les sentiments, les passions ou les humeurs en les classifiant, en les étendant sans qu'il soit possible de s'accorder aujourd'hui sur une définition unanimement reconnue ${ }^{26}$.

\subsection{L'émotion et la raison : deux grilles de lecture d'une même réalité}

L'émotion ne s'oppose pas frontalement à la rationalité mais tisse des liens étroits avec elle: loin d'interférer, elle pourrait aider à prendre rationnellement une décision dans les situations d'incertitude par exemple ${ }^{27}$. L'émotion peut également être qualifiée de rationnelle ${ }^{28}$. Il s'ensuit qu'il est dès lors plus indiqué d'imaginer des grilles de lecture différente d'une même réalité : il est extrêmement raisonnable de rouler à droite sur les routes continentales alors qu'une lecture émotionnelle de la question insisterait sur la crainte d'un accident (sans qu'il soit par ailleurs besoin d'invoquer celle d'une sanction pénale); la théorie des choix rationnels peut conduire à préférer une solution impliquant de violer délibérément la loi si celle-ci coûte moins cher à l'infracteur tant financièrement qu'en termes d'image comme les études en law and economics l'ont théorisé ${ }^{29}$, alors qu'une lecture émotionnelle montrerait que le désir de richesse, l'appât du gain, la cupidité ou la peur de l'appauvrissement pèseraient plus que la fierté et l'honneur de respecter la loi ou l'embarras et la culpabilité que sa violation pourrait susciter. L'opposition entre raison et émotion est en réalité utilisée à des fins persuasives dans tout processus de décision, que celui-ci soit juridique, politique, économique ou autre: celui qui appelle l'autre à la raison tente en fait de lui montrer qu'il existe d'autres émotions susceptibles de fonder sa décision qui doivent l'emporter ou que certaines émotions peuvent être apaisées. Les décisions qualifiées d'émotionnelles illustrent le propos. L'internement à vie des délinquants dangereux est émotionnellement fondé sur le dégoût et la peur du criminel pervers, sur l'empathie envers les victimes et la tristesse ainsi que la colère que celles-ci peuvent ressentir; les mesures restrictives à l'égard des étrangers sont essentiellement fondées sur la peur de l'autre en raison de la dangerosité prétendue ou de la crainte

${ }^{26}$ Voir Deonna, Julien/ Teroni, Fabrice, Qu'est-ce qu'une émotion? Paris 2008.

${ }^{27}$ Elster 1999 [note 48], p. 283ss; Damasio, Antonio, L'erreur de Descartes: la raison des émotions, Paris 1999 (nouvelle éd. 2008); Scherer, Klaus, Zur Rationalität der Emotionen, in Rössner, H. (éd.), Der ganze Mensch, p.181ss, Munich 1986.

${ }^{28}$ Bandes, Susan, The Passion of Law, New York, 2000, p. 10; Elster 1999 [note 48], p. 284ss.

${ }^{29}$ Voir en particulier l'analyse économique de la délinquance selon laquelle le délinquant est supposé agir «rationnellement» lorsqu'il commet un acte répréhensible en comparant le bénéfice que lui rapporte cet acte à son coût (montant de la sanction pécuniaire, équivalent monétaire d'une peine privative de liberté): Becker, G.S., «Crime and Punishment: An Economic Approach», Journal of Political Economy 1968, p. 1ss; Garoupa, Nuno, «The Theory of Optimal Law Enforcement», Journal of Economic Surveys 1997, 11, p. 267ss; Polinsky, Mitchell/ Shavell, Steven, «The Economic Theory of Public Enforcement of Law » Journal of Economic Literature, American Economic Association 2000, 38, p. 45ss. En droit de l'environnement, voir Hiriart, Yolande, «L'utilisation du droit pénal en matière environnementale,» IDEI Working Papers 326, Institut d'Économie Industrielle (IDEI), Toulouse 2004. Pour une approche comportementale de l'analyse économique du droit, voir Sunstein, Cass, Behavioral law and economics, Cambridge [etc.] 2000. 
que les indigènes perdent leurs emplois. Auprès de certains, ces émotions sont prépondérantes et l'emportent définitivement; chez d'autres elles sont contrebalancées par des émotions soit semblables, mais dirigées autrement telles que l'empathie et la pitié envers le coupable, soit différentes tels que la joie de l'ouverture au monde ou le désir, plus calculateur (mais certainement pas plus «rationnel»), de disposer d'une main d'œuvre à bon marché.

Le propos ne consiste ainsi pas à opposer raison et émotion, mais à sonder la variable émotionnelle dans les mécanismes à l'œuvre dans le respect des règles. Cette perspective est évidemment limitative, mais nous paraît opportunément compléter les analyses de type politique, sociologique ou économique, plus traditionnelles.

\subsection{L'émotion au service du respect du droit, hard et soft}

Pour améliorer l'observation de la soft law, l'Etat peut faire appel à diverses techniques de persuasion en sollicitant plus ou moins fortement le registre émotionnel. Cette démarche n'est en soi pas originale dans la mesure où le rôle des émotions peut facilement être mis en évidence dans la mise en œuvre du droit classique: le droit pénal, dans sa composante punitive primitive (confinement, exclusion, appauvrissement, humiliation, souffrance ou mort), est conçu pour inspirer peur ${ }^{30}$, angoisse ou terreur dans le grand public, générer honte, embarras ou culpabilité auprès des coupables et calmer l'esprit de vengeance, de colère ou de rage des victimes afin d'imposer le respect des règles de droit. Les justiciables obéissent à la loi en raison d'un lien émotionnel comprenant pour certains la crainte de la punition ou de la honte qui s'ensuit ${ }^{31}$ ainsi que, pour d'autres, des émotions positives telles que le respect ou l'engagement envers le bien public ${ }^{32}$. La peur de la sanction n'est toutefois de loin pas le seul facteur explicatif ${ }^{33}$.

On notera, s'agissant de la hard law, que la place des émotions a déjà été examinée plus largement, que ce soit au niveau de la décision judiciaire ${ }^{34}$, du

${ }^{30}$ Lange, Bettina, «The Emotional Dimension in Legal Regulation », Journal of Law and Society, mars 2002, p. 197ss, 209.

${ }^{31}$ Miller, William, «Fear, Weak Legs, and Running Away: A Soldier's Story », in Bandes, Susan (éd.), The Passion of Law, New York, 2000, p. 242. Voir ég. ci-dessous ch. 2.3.3.

${ }^{32}$ Deigh, John, «Emotion and the Authority of Law», in Bandes, Susan (éd.), The Passion of Law, New York, 2000, p. 285ss.

${ }^{33}$ Voir ci-dessous ch. 2.3.3.

${ }^{34}$ Noll, Andreas, «Vom abnehmenden Grenznutzen des Justizsystems », Revue de droit suisse I, 2007 , p. 572 pour lequel la décision judiciaire est un pur processus émotionnel; seules les phases qui précèdent (choix des alternatives de décision) et qui suivent (justification du choix) seraient rationnelles. Noll insiste sur la nécessaire empathie du juge pour réduire la complexité (p. 571ss. Plus sceptique sur les qualités d'empathie du juge en en mettant les dangers en évidence: Bandes, Susan, The Passion of Law, New York, 2000, p. 9). Voir ég. Van den Bos, K., «On the subjective quality of social justice: The role of affect as information in the psychology of justice judgments », Journal of Personality and Social Psychology, 85, pour qui, particulièrement en cas d'incertitude, le juge devant trancher sera amené à percevoir la situation à juger de façon plus positive ou négative selon son état émotionnel.

La persuasion des juges par des procédés de rhétorique a été débattue dès l'antiquité: Artistote considérant au contraire de Cicéron qu'il ne faut pas éveiller chez le juge des sentiments susceptibles 
processus législatif ${ }^{35}$, en rapport avec le sentiment de justice ${ }^{36}$, le libre arbitre et l'émotion dans les jugements moraux ${ }^{37}$, le rôle des avocats ${ }^{38}$, celui des recourants $^{39}$, celui du jury populaire ${ }^{40}$ ainsi qu'en lien avec les émotions éprouvées par

d'interférer avec l'évaluation objective du cas (réf. cit. in Ruth Amossy, L'argumentation dans le discours, Paris 2000 (2006 2 éd), p. 166). Voir ég. Scherer, Klaus, «Emotion und Propaganda: Zur Psychologie des Pathos», NZZ, édition du 29-30 septembre 1989, p. 25.

Pour certains réalistes américains tels Jerome Frank, les explications psychologiques et émotionnelles du juge seraient déterminantes dans la décision de justice: la théorie dite du «petit-déjeuner» postule que le petit-déjeuner du juge influence son appréciation du cas d'espèce. La connaissance du droit découle alors de la connaissance de la composition du petit-déjeuner du juge et de l'impact sur ses émotions (cit. in Millard, Eric, Théorie générale du droit, Paris 2006, p. 56 et 100; lequel propose une reformulation de la théorie de Frank, p. 109ss).

Pour Bandes, il est dangereux, tant pour le juge que pour l'ordre juridique lui-même, que de nourrir l'illusion selon laquelle le juge opèrerait dans un champ libre d'émotions (Bandes, Susan, The Passion of Law, New York, 2000, p. 6s). Voir ég. Löschper, Gabrielle, Bausteine für eine psychologische Theorie richterlichen Urteilens, Baden-Baden, 1999; Weimar, Robert, Psychologische Strukturen richterlicher Entscheidung, Bern 1996; Ludewig-Kedmi, Revital, „Moraldilemmata von Richtern«, Ehrenzeller, Bernhard / Ludewig-Kedmi, Revital, Moraldilemmata von Richtern und Rechtsanwälten, Saint-Gall 2006, p. 9ss (p. 12ss sur la place de la psychologie dans la décision judiciaire); Schubarth, Martin, «Der Richter zwischen Rationalität und Sensibilität», Recht 1995, p. 151 ss.

${ }^{35}$ Lange, Bettina, «The Emotional Dimension in Legal Regulation », Journal of Law and Society, mars 2002,p. 204ss, p. 206ss. Sur le rôle joué tant par le dégoût que l'amour romantique dans la genèse des lois en faveur des homosexuels, voir Calhoun, Cheshire, «Making Up Emotional People: The Case of Romantic Love», in Bandes, Susan (éd.), The Passion of Law, New York, 2000, p. 217ss. Sur le rôle crucial joué par le dégoût dans la genèse des lois discriminant les homosexuels, les femmes, les juifs et autres marginaux, voir Nussbaum, Martha, «Secret Sewers of Vice»: Disgust, Bodies, and the Law, in Bandes, Susan (éd.), The Passion of Law, New York, 2000, p. 19ss.

${ }^{36}$ Solomon, Robert, «The emotions of justice», Social Justice Research, vol. 3, no 4, 1989, p. 345 ss.

${ }^{37}$ Greene, J. D./ Cohen J. D., «For the law, neuroscience changes nothing and everything » Philosophical Transactions of the Royal Society of London B (Special Issue on Law and the Brain), 2004, 359 p. 1775ss. Critique à cet égard: Papaux, Alain, «Détermination biologique des comportements et responsabilité individuelle: une approche de la philosophie du droit, in Morale et évolution biologique: entre déterminisme et liberté, Lausanne 2007, p. 295ss. Voir ég. Rainer, Silvie/ Fäh, Luzia/ Killias, Martin, „Freier Wille oder Zwang?: zur Ausklammerung probabilistischer Zusammenhänge und situativer Einflüsse in neurowissenschaftlichen Ansätzen«, Schweizerische Zeitschrift für Kriminologie, 2007, 1, p. 3ss.

${ }^{38}$ Un métier, s'agissant des avocats pratiquant le barreau, impliquant de montrer ou de provoquer des émotions négatives chez autrui en étant dur, agressif et intimidant (Pierce J. L., Gender Trials. Emotional Lives in Contemporary Law Firms, Berkeley 1995, mettant en évidence les différences liées au sexe).

${ }^{39}$ Solomon, Robert, «Justice v. Vengeance: On Law and the Satisfaction of Emotion», Susan (éd.), The Passion of Law, New York, 2000, p. 123ss, met en évidence le rôle de la colère, du ressentiment et de la vengeance dans les motivations de celui qui ouvre action.

${ }^{40}$ Ellsworth, Phoebe, «Some Steps between Attitudes and Verdicts», in Hastie, R., Inside the Juror: The Psychology of Juror Decision Making, New York 1993; Bartol, Curt/ Bartol, Anne, Psychology and Law: Research and Application, 2e éd., Pacific Grove 1994, p. 6 s. 
les parties au procès ${ }^{41}$ ou les victimes ${ }^{42}$. Certains travaux sont basés sur des émotions spécifiques en rapport avec le droit (bonheur ${ }^{43}$, douleur ${ }^{44}$, peur $^{45}$ ).

Dans le contexte de la soft law, les travaux sur l'obéissance aux normes sociales $^{46}$ et sur les techniques de persuasion ${ }^{47}$ sont d'un grand intérêt. L'observation des règles non obligatoires ne saurait s'expliquer uniquement d'après la théorie des choix rationnels mais reposerait essentiellement sur des mécanismes de nature émotionnelle, en particulier l'anticipation de la honte, de la culpabilité ou l'embarras en cas de violation ainsi que la fierté en cas de respect ${ }^{48}$; la honte étant provoquée par le dégoût ou le mépris que les autres éprouvent à l'égard du violateur $^{49}$.

La lecture émotionnelle n'est ainsi qu'une lecture possible des mécanismes de respect des règles. Même si elle possède un fort potentiel explicatif à la question de savoir pourquoi nous modifions nos attitudes ${ }^{50}$, il faut être conscient de l'existence d'autres leviers qu'une approche exclusivement émotionnelle n'expliquerait que partiellement. C'est pourquoi nous examinerons dans une seconde partie les techniques de manipulation, amenant les individus à modifier leur comportement à leur insu. On doit également signaler les recherches relativisant l'effet de l'émotion (en l'espèce la peur de la punition) dans le respect de la loi. Selon ces travaux, on obéit à la loi si les destinataires croient en sa légitimité ${ }^{51}$ - définie de manière procédurale ${ }^{52}$ - en s'y conformant spontanément ${ }^{53}$. Les explications

${ }^{41}$ Minow, Martha, «Institutions and Emotions: Redressing Mass Violence», in Bandes, Susan (éd.), The Passion of Law, New York, 2000, p. 265ss, souligne que les procès en divorce exacerbent l'amertume plus qu'ils ne la canalisent.

${ }^{42}$ Robert, Christian-Nils, «Autour des victimes: pensée unique et pensée critique», Aide aux victimes, Médecine et hygiène 1997, p. 323ss; Bandes, Susan, «Empathy, Narrative and Victim Impact Statements », University Chicago Law Review 1996, p. 361ss.

${ }^{43}$ Rehbinder, Manfred/ Usteri, Martin (éd.), Glück als Ziel der Rechtspolitik, Berne 2002.

44 Durand, Bernard/ Poirier, Jean/ Royer Jean-Pierre, La douleur et le droit, Paris 1997.

${ }^{45}$ Sunstein, Cass, Laws of fear: beyond the precautionary principle, Cambridge [etc.] 2005.

${ }^{46}$ Posner, Eric, Law and Social Norms, Cambridge Mass 2000; Posner, Eric (éd.), Social Norms, Nonlegal Sanctions, and the Law, Cheltenham, Northampton 2007; O’Donnell, Patrick, «Social Norms \& Law: An Introduction», Theory \& Science 2007, vol. 9/2.

${ }^{47}$ Petty, R./ DeSteno, D./ Rucker, D., «The role of affect in attitude change», Forgas, Joseph (éd.), Handbook of affect and social cognition, Mahwah 2001, p. 212ss; Petty, Richard/ Gleicher, Faith/ Baker, Sara, «Multiple roles of affect in persuasion », Forgas Joseph (éd.), Affect and Judgment, London 1991, p. 181ss.

${ }^{48}$ Elster, Jon, Alchemies of the Mind: Rationality and the Emotions, Cambridge 1999, p. 145ss. Pour Gagné (Gagné, Learry, «Non-rational compliance with social norms : sincere and hypocritical», Social Science Information 2007, 46, p. 445ss) s'inspirant de Pierre Bourdieu, le conformisme non rationnel serait mieux accepté par le groupe que le conformisme intéressé.

${ }^{49}$ Elster 1999 [note 48], p. 149.

50 Voir réf. ci-dessus note 47.

51 Tyler, Tom, Why People Obey the Law, Princeton 2006 (2e éd.).

52 Tyler 2006 [note 51], p. 272.

${ }^{53}$ Killias, Martin, Précis de criminologie, $2^{\mathrm{e}}$ éd., Berne 2001, p. 457s et 467ss pour lequel la peur du gendarme est globalement moins importante que le conformisme spontané de la plupart des gens dans le respect des lois. 
morales, éthiques ou en termes de justice trouvent dans ce type de travaux un fondement bienvenu: je ne tue personnellement pas d'autres êtres humains, non pas par peur de la sanction pénale, mais simplement par conviction intime de la justesse, de la justice et de la légitimité de cette règle. Il ne sert dès lors à rien au législateur de s'engager dans une escalade de mécanismes de mise en œuvre distillant la peur alors qu'il devrait plutôt œuvrer à rendre le système légal plus digne de respect en travaillant sur sa légitimité.

\subsection{Les ressorts émotionnels du respect de la soft law}

\subsubsection{Introduction: l'effet non obligatoire de la soft law}

Ne revêtant pas la forme de la norme juridique, la soft law est en soi dénuée d'effets obligatoires. Elle a pour effet d'orienter le comportement de ses destinataires sans les obliger juridiquement. Elle conserve pourtant son caractère normatif, car la norme n'est pas nécessairement impérative. Il faut en effet éviter de confondre la normativité avec l'impérativité: «Le normatif est un genre qui contient deux espèces principales: l'impératif et l'appréciatif ${ }^{55}$. Le comportement des destinataires de la soft law est laissé à leur propre appréciation ${ }^{56}$. La soft law présente donc une nature normative appréciative et non impérative. Elle ressortit non pas au «Sollen» (devoir-être) mais au «Sollten» (devrait-être) ${ }^{57}$.

Dans certaines circonstances toutefois, la soft law est susceptible de déployer de véritables effets juridiques obligatoires. Tel est le cas lorsqu'un renvoi législatif ou judiciaire confère directement à une recommandation une force contraignante; l'effet obligatoire ne découle ici pas de la recommandation mais de la loi qui y renvoie ${ }^{58}$. Le lien peut aussi être indirect lorsque la recommandation, sans être impérative pour les administrés, est obligatoire pour les autorités chargées de l'appliquer ${ }^{59}$. La soft law peut enfin avoir une valeur permissive en ce sens que celui qui suit une recommandation étatique ne saurait être actionné en responsabilité pour avoir causé un dommage parce qu'il l'a suivie ${ }^{60}$. Le mécanisme émotionnel du respect de ces actes dans ces hypothèses n'est pas différent, sur le principe, de celui des actes juridiques obligatoires.

En revanche lorsque la soft law ne présente qu'un pur effet appréciatif, l'examen des ressorts émotionnels de son observation doit être approfondi puisque les destinataires sont libres de s'en détourner. Les mécanismes peuvent être purement factuels en ce sens qu'ils exercent par eux-mêmes une influence parfois importante. L'ordre juridique peut également instaurer divers dispositifs - juridiques misant en particulier sur une exacerbation de l'émotion afin d'accroître le respect de la soft law.

${ }^{54}$ Tyler 2006 [note 51], $4^{\mathrm{e}}$ de couverture.

55 Lalande, André, Vocabulaire technique et critique de la philosophie, Paris 1993 (1 1 ère édition 1926), sous l'entrée «normatif». Voir ég. Amselek 1991, p. 143.

${ }^{56}$ Flückiger 2004 [note 11], p. 230.

${ }^{57}$ Flückiger 2004 [note 11], p. 190.

${ }^{58}$ Flückiger 2004 [note 11], p. 237.

${ }^{59}$ Flückiger 2004 [note 11], p. 240ss.

${ }^{60}$ Flückiger 2004 [note 11], p. 246. 


\subsubsection{Le respect de l'autorité}

Le premier effet découle de l'ascendant ou du prestige lié à la figure de l'auteur et des émotions ainsi suscitées (crainte diffuse, désir de plaire ou de reconnaissance, admiration). La recommandation émanant d'une autorité publique a plus d'influence que le même conseil émanant d'un quidam ou d'une obscure association. Tel est le cas par exemple des recommandations de la Commission fédérale de la concurrence ${ }^{61}$ ou celles du Surveillant des prix, lequel a «au moins un certain poids moral» ${ }^{62}$.

L'ascendant de l'auteur lié au contexte peut également avoir une visée «performative» pour reprendre la terminologie de la théorie des actes de langage en linguistique ${ }^{63}$. Un énoncé a priori descriptif devient une recommandation d'autant plus forte qu'elle émane d'une autorité reconnue dans un contexte spécifique: lorsque le constat scientifique des effets de la fumée sur l'organisme humain selon lequel «fumer tue» est diffusé dans le contexte particulier d'une campagne de prévention étatique ou sur un paquet de cigarette, il est spontanément lu comme une recommandation de ne pas fumer ${ }^{64}$.

Un pari est fait sur l'ascendant de l'auteur lorsque les autorités adoptent délibérément un comportement exemplaire dans le cadre d'une politique publique (trier les déchets, rénover les bâtiments publics pour procéder à des économies d'énergie), proposant un modèle à suivre auprès des particuliers et les habituant dans l'espoir de les entraîner. La publicité que donne l'Etat à son nouveau comportement sonne auprès des administrés comme une véritable recommandation d'adopter la même attitude que lui ${ }^{65}$.

Les travaux en psychologie sociale relatifs à l'obéissance montrent l'importance du poids qu'accordent les individus à une figure d'autorité ${ }^{66}$, indépendamment de l'existence d'un lien juridique obligatoire ${ }^{67}$. Les sources crédibles sont plus convaincantes. L'impact de l'influence des experts a par exemple pu être démontré dans le cadre des campagnes anti-tabac ${ }^{68}$.

${ }^{61}$ Tercier, Pierre, $a d$ art. 45 LCart, ch. 45, p. 948 et Martenet, Vincent, ad art. 8 LMI, ch. 8, p. 1392, in Tercier, P.; Bovet, C. (éd.), Droit de la concurrence: loi sur les cartels, loi sur la surveillance des prix, loi sur le marché intérieur, loi sur les entraves techniques au commerce: commentaire, Genève, etc. 2002.

${ }^{62}$ Bonvin, Jacques, ad art. 14 LSPr, ch. 40, p. 1172, in Tercier, P.; Bovet, C. (éd.), Droit de la concurrence: loi sur les cartels, loi sur la surveillance des prix, loi sur le marché intérieur, loi sur les entraves techniques au commerce: commentaire, Genève, etc. 2002.

63 Austin, John Langshaw, Quand dire c'est faire (trad. par G. Lane), Paris, Seuil, 1970.

${ }^{64}$ La description est «incitatrice» (voir Flückiger 2004 [note 11], p. 203).

${ }^{65}$ Flückiger 2004 [note 11], p. 201s.

${ }^{66}$ Girandola, Fabien, «La source», in Girandola, Psychologie de la persuasion et de l'engagement, Besançon 2003, p. 17ss.

${ }^{67}$ L'expérience de Milgram poussant les sujets à obéir à des ordres inhumains doit se comprendre dans un cadre de hard law dans la mesure où l'expérimentateur leur donnait des commandements directifs (Milgram, Stanley, «Behavioral Study of Obedience», Journal of Abnormal and Social Psychology 67, 1963, p. 371ss).

${ }^{68}$ Falomir Pichastor, Juan Manuel/ Mugny, Gabriel, Société contre fumeur: une analyse psychosociale de l'influence des experts, Grenoble, 2004. 
Certains actes non obligatoires sont adoptés, rédigés et formulés comme des actes obligatoires, induisant un effet de bluff auprès des destinataires ${ }^{69}$. L'obéissance se produit ici par décalque en quelque sorte, «fondant des espoirs secrets sur la force normative de l'écrit» ${ }^{70}$ ou impressionnant par la solennité de la procédure d'adoption à l'instar de certaines déclarations internationales sans valeur obligatoire $^{71}$ telles la Déclaration de Rio (environnement) ou celle de Bologne (éducation). Un auteur a ainsi pu écrire à propos des actes non juridiquement obligatoires, en généralisant la réflexion à partir de l'exemple de la Charte européenne des droits de l'homme dans la ville, que «contraignant par la persuasion et par l'incitation, [ces actes] peuvent conduire à intérioriser des pratiques voulues par l'administration, parfois même sur la base d'une croyance en des sanctions potentielles même si celles-ci ne sont pas formulées, sans qu'aucun juge ne vienne protéger la liberté individuelle et anéantir ce pouvoir de contrainte non juridique qui se situe en deçà de sa compétence. Tout acte informel met ainsi en place une apparence de pouvoir, un simulacre de commandement que peu d'administrés sont à même de contester au nom de la réalité de leurs droits.» ${ }^{72}$

Dans d'autres cas, le respect de l'autorité est stratégique. Il peut découler de la nécessité de créer un climat de confiance en entretenant de bonnes relations avec l'administration, condition déterminante dans le domaine des arrangements non obligatoires (gentlemen's agreement) ${ }^{73}$. Ici la crainte d'une détérioration des rapports pousse à obéir aux actes non obligatoires. Cette motivation est particulièrement forte en droit international public où la nécessité de maintenir de bonnes relations entre Etats a pour conséquence que la violation d'un engagement purement politique peut engendrer des mesures de rétorsion diverses ${ }^{74}$.

Cet effet va si loin que le législateur lui-même se sent parfois lié par la soft law, politiquement tout au moins. Le Conseil fédéral écrit par exemple, à propos du projet de recommandation du Comité des ministres du Conseil de l'Europe sur le financement des partis politiques et des campagnes électorales, que «si cette recommandation devait être adoptée, il faudrait alors procéder en Suisse également à un nouvel examen de toute une série de questions, en tenant compte de particularités institutionnelles telles que le gouvernement collégial, la démocra-

${ }^{69}$ Flückiger 2004 [note 11], p. 247.

${ }^{70}$ Tschannen, Pierre, Der Richtplan und die Abstimmung raumwirksamer Aufgabe, Berne, let. e, p. 325 .

${ }^{71}$ Nguyen Quoc Dinh/ Daillier, Patrick/ Pellet, Alain, Droit international public, $7^{\mathrm{e}}$ éd. Paris 2002, ch. 247, p. 378.

${ }^{72}$ Chabrot, Christophe, «La charte européenne des droits de l'homme dans la ville: un exemple d'acte 'pré-juridique'?», Revue de Droit Public, 2007, 2, p. 355ss, 375.

${ }^{73}$ Kautz, Steffen, Absprachen im Verwaltungsrecht: Zulässigkeit, Grenzen und Folgen, Berlin 2002, p. 80s; Müller-Graf, Thomas, Entrechtlichung durch Informalisierung? ein Beitrag zur Handlungsformen- und zur Rechtsverhältnislehre im Verwaltungsrecht, Bâle, etc. 2001, p. 173s.

${ }^{74}$ Virally, Michel, «La distinction entre textes internationaux de portée juridique et textes internationaux dépourvus de portée juridique (à l'exception des textes émanant des organisations internationales): rapport provisoire», Annuaire de l'Institut de droit international, vol. 60, tome I, Paris 1983 , p. 231 s. 
tie semi-directe et le fédéralisme.» ${ }^{75}$ Le programme de législature et de la politique extérieure, et plus généralement les arrêtés relatifs aux planifications, comme le souligne la Commission des institutions politiques du Conseil national, «sans avoir valeur de règle de droit, n'en ont pas moins une portée politique considérable, puisqu'elles influent de manière déterminante sur l'issue $d u$ processus ${ }^{76}$. En droit international public, «les recommandations peuvent être politiquement très contraignantes. Ce sont d'indéniables moyens de pression politiques.» ${ }^{77}$ La technique de la loi-modèle et des traités-types par laquelle une autorité supérieure propose de suivre un modèle de loi afin d'uniformiser une pratique dans les domaines où elle n'en dispose pas forcément de la compétence ${ }^{78}$ repose également sur un fondement psychologique analogue.

\subsubsection{La peur}

\subsubsection{L'influence directe}

La peur, la crainte, l'angoisse ou l'anxiété sont de puissants vecteurs d'obédience. Il suffit que l'Etat les actionne dans des instruments de soft law pour que le public-cible soit fortement incité à adapter son comportement. S'il est vrai qu'une peur accrue augmente généralement l'aspect persuasif du message, les psychologues et les criminologues, notamment, se sont toutefois efforcés d'en montrer les limites ${ }^{79}$.

La peur peut être liée à d'autres émotions négatives qui en renforcent l'impact telles que le dégoût, l'aversion, l'indignation, la colère ou la tristesse: la peur des conséquences du réchauffement climatique peut rendre triste ou provoquer la colère; dans les campagnes anti-tabac, la crainte de la maladie peut en effet être renforcée par des images inspirant le dégoût ${ }^{80}$, etc. Ces autres émotions négatives peuvent évidemment influencer le comportement par elles-mêmes : c'est le simple dégoût provoqué à la vue des crachats sur le sol qui me retient de cracher et pas forcément la crainte d'un regard réprobateur ou d'une autre sanction sociale; c'est ma propre aversion envers des comportements heurtant ma sensibilité et mon sentiment de justice et d'équité qui me retient de les adopter.

${ }^{75}$ Conseil fédéral, Réponse du 20 février 2002 à l'interpellation Maillard (01.3767 Argent et démocratie. Liaisons dangereuses).

${ }^{76}$ Loi sur le Parlement: rapport [...] du $1^{\text {er }}$ mars 2001, FF $20013298,3322$.

${ }^{77}$ Nguyen 2002 [note 71], ch. 247, p. 378. Voir dans le même sens, Virally, Michel, «La distinction entre textes internationaux de portée juridique et textes internationaux dépourvus de portée juridique (à l'exception des textes émanant des organisations internationales): rapport provisoire », Annuaire de l'Institut de droit international, vol. 60, tome I, Paris 1983, p. 233, pour qui les engagements purement politiques sont parfois davantage contraignants que des engagements juridiques.

78 Voir Flückiger 2004 [note 11], p. 200s.

79 Petty et al. 2001 [note 47], p. 217ss; Leventhal, H., «Findings and theory in the study of fear communications», Berkowitz, L. (éd.), Advances in experimental social psychology, vol. 5., 1970, p. 120ss. Voir ég. la thèse de Tyler 2006 [note 51] et Killias 2001 [note 53].

${ }^{80}$ Voir la reproduction des images autorisées à l'annexe 1 de l'ordonnance du Département fédéral de l'intérieur concernant les mises en garde combinées sur les produits du tabac (RS 817.064). 
Tel est le cas de nombreuses campagnes d'information en matière de santé publique: la peur de la mort, de la maladie, de la déchéance ou de la honte dans le cadre de la lutte contre le sida; la peur de la pénurie d'organes dans le cas des campagnes de promotion du don d'organe; la peur du cancer dans les campagnes anti-tabac; la peur de la mise en danger de la santé des personnes âgées, des malades et des bébés dans les campagnes d'information en période de canicule; la peur de la mort des forêts ou du réchauffement climatique dans les campagnes en faveur de la protection de l'environnement ${ }^{81}$; la peur de l'accident dans les campagnes de sensibilisation à la sécurité routière ou en entreprise; la peur de la maladie dans les campagnes de prévention de l'obésité; la peur de l'ennemi dévoilant sa férocité dans la propagande militaire ${ }^{82}$, etc.

Ces craintes primaires ne sont en réalité pas les seules émotions qui conduisent à suivre les comportements préconisés par de telles campagnes. La réalité est plus enchevêtrée. L'efficacité doit être lue en combinaison avec d'autres outils, y compris avec les actes juridiques. L'effet des campagnes d'information anti-tabac ou antialcoolique est ainsi renforcé par des taxes spéciales perçues sur les paquets de cigarette ou sur l'alcool fort ${ }^{83}$. Des motivations parallèles se rencontrent parfois, à l'instar du taux de rapports sexuels protégés dont une part peut être imputée directement aux campagnes antisida, mais dont l'autre peut provenir de motifs concurrents tels que l'anxiété d'une grossesse indésirable, la crainte d'autres maladies sexuellement transmissibles ou la crainte d'une sanction pénale.

Le recours aux images dans de telles campagnes en augmente incontestablement l'impact émotionnel comme les exemples de la lutte anti-tabac ou de la prévention des accidents de la route le montrent. L'efficacité de l'influence des images en contexte de propagande est néanmoins aujourd'hui controversée ${ }^{84}$.

Les mises en garde sont des recommandations particulières portant sur la dangerosité de personnes, de produits ou de comportements et se manifestent généralement sous la forme d'invitation à s'abstenir ${ }^{85}$. Elles inspirent par définition la peur en prévenant du danger. On en trouve dans le domaine des médicaments ${ }^{86}$, des

${ }^{81}$ Certains philosophes en ont même déduit une «heuristique» (voir l'heuristique de la peur chez Hans Jonas, Le principe responsabilité: une éthique pour la civilisation technologique, Paris 2003 [trad.] [1979]).

${ }^{82}$ Géré, François, La guerre psychologique, Paris, 1997.

${ }^{83}$ Voir par exemple l'effet de la taxe sur les alcopops sur la consommation de telles boissons (Feuille fédérale 2003 1984).

${ }^{84}$ A l'origine des théories de l'efficacité de telles images, voir Tchakhotine, S., Le viol des foules par la propagande politique, 1939, rééd., Paris, 1992; Ellul, Jacques, Propagandes, Paris, 1990. Contra: Gervereau, L., Histoire du visuel au XXe siècle, Paris, 2003.

${ }^{85}$ Tschannen, Pierre, «Amtliche Warnungen und Empfehlungen», Revue de droit suisse II 1999, p. 365s; Flückiger 2004 [note 11], p. 193ss.

${ }^{86}$ Le public doit être informé «des événements particuliers en relation avec les produits thérapeutiques présentant un danger pour la santé, et [1'Institut suisse des produits thérapeutiques veille] à ce que des recommandations sur le comportement à adopter soient émises» (art. 67 de la loi fédérale sur les médicaments et les dispositifs médicaux [RS 812.21]). 
jouets $^{87}$, des denrées alimentaires ${ }^{88}$, de l'énergie nucléaire ${ }^{89}$, des croyances religieuses ${ }^{90}$ ou des avalanches ${ }^{91}$.

Enfin, plus généralement, la pression de changement peut provenir du groupe social auquel le destinataire appartient, si ce dernier craint par exemple d'entrer en conflit avec le groupe. Les psychologues sociaux qualifient cette attitude de conformisme $e^{92}$.

Le regret est également à l'œuvre dans les mécanismes d'obéissance, plus particulièrement la peur anticipée du regret ou du remord qu'une conduite contraire à une recommandation pourrait nous valoir. Les économistes en ont même proposé une théorie: la «théorie des regrets ${ }^{93}$, selon laquelle la peur de regretter quelque chose - par exemple pour un investisseur celle de rater une hausse future ou de subir une perte plus tard - conduit à des décisions peu rationnelles. Tel sera l'effet du regret ou du remords rétrospectif de ne pas avoir porté un préservatif après un acte sexuel risqué ou du remords de ne pas avoir voulu signer sa carte de donneur d'organe au moment où l'on aura soi-même besoin d'être transplanté.

\subsubsection{Les mécanismes juridiques de recours à la peur dans les instruments de soft law}

L'ordre juridique peut prévoir divers mécanismes, juridiques, destinés à renforcer l'observance des règles de soft law. Celles-ci ne deviennent pas pour autant juridiquement obligatoires. Pourtant les mécanismes psychologiques que le droit provoque peuvent être de nature analogue à ceux des actes obligatoires. La

${ }^{87}$ Annexe III ch. II de l'ordonnance du DFI sur la sécurité des jouets (RS 817.044.1). Voir par exemple OFSP, Mise en garde concernant un jouet: danger d'étranglement avec les yoyo balls ou «balles hérissons», Berne, 26 mars 2003.

${ }^{88}$ L'autorité compétente à mettre en garde publiquement la population contre des denrées alimentaires, des additifs ou des objets usuels présentant un danger pour la santé et qui ont été distribués à un nombre indéterminé de consommateurs; l'autorité informe dans ce cas le public et lui recommande le comportement à adopter (art. 43 al. $1^{\text {er }}$ LDAl [RS 817.0]). Voir par exemple une mise en garde relative à la consommation d'un fromage au lait cru, le vacherin Mont d'Or (Arrêt du Tribunal fédéral 118 Ib 473).

${ }^{89}$ Mise en garde concernant la consommation de salade en Suisse à la suite de l'accident de la centrale nucléaire de Tchernobyl (Arrêt du Tribunal fédéral 116 II 480).

90 Voir les cas de l'association infoSekta (Arrêt du Tribunal fédéral 118 Ia 46) et de la publication du livre «Das Paradies kann warten» (Arrêt du Tribunal fédéral 121 I 87), décrits in Tschannen, Pierre, «Amtliche Warnungen und Empfehlungen», Revue de droit suisse II 1999, p. 370s.

${ }^{91}$ L'Institut fédéral de recherches sur la forêt, la neige et le paysage a pour tâche d'assurer le service de mise en garde contre les avalanches en Suisse et l'information du public sur le danger d'avalanches (art. 4 al. 4 de l'ordonnance sur le FNP [RS 414.164]).

92 Asch, S. E., «Effects of group pressure upon the modification and distortion of judgment», H. Guetzkow (éd.) Groups, leadership and men. Pittsburgh 1951; Doise, W., Deschamps, J.-C. et Mugny, G., Psychologie sociale expérimentale, 2e éd., Paris, A. Colin, 1991, chapitre 7. Sur le rôle du conformisme dans le respect de la soft law dans le domaine financier, voir Roth, Monika, Soft law: Ordnungsvisionen in flux: eine Standortbestimmung mit Fokus auf compliancerelevante Fragestellungen, Berne 2006, p. 89.

93 Loomes, Graham/ Sugden, Robert. «Regret Theory: An Alternative Theory of Rational Choice Under Uncertainty», The Economic Journal, vol. 92, no 368, 1982, p. 805ss. 
distinction entre les sanctions juridiques et les «pressions » tendant à la réalisation de conduites non obligatoires est extrêmement ténue ${ }^{94}$.

Tel est le cas de la pression financière qui se cache derrière les taxes incitatives. Celles-ci contiennent des recommandations non obligatoires, doublées juridiquement du paiement d'une somme d'argent. Elles comprennent une double composante, impérative et non impérative. Le comportement préconisé n'est pas obligatoire, mais simplement recommandé. Cet instrument peut cependant être compris par ses destinataires comme une sanction pécuniaire très semblable aux sanctions juridiques ordinaires: celui qui ne trie pas ses déchets doit s'acquitter d'une taxe $\mathrm{e}^{95}$. La différence tient au fait que dans la variante recommandationnelle, l'acquittement d'une taxe n'a pas la même portée symbolique que dans la variante pénale sanctionnée par une peine pécuniaire. La crainte de l'appauvrissement ou le désir de ne pas perdre de l'argent, générés dans les deux hypothèses, sont doublés dans la seconde variante de l'anxiété propre à l'ouverture d'une procédure pénale corrélée symboliquement à des sentiments de honte et de culpabilité. En pratique toutefois la différence tend à s'estomper chaque fois que de faibles sommes sont en cause, dans la mesure où la procédure pénale est alors simplifiée. La sanction est perçue dans ce cas comme une $\left\langle\right.$ simple taxe» ${ }^{96}$ ! Les frontières de la notion même de sanction deviennent incertaines en présence de la soft law ${ }^{97}$; elles s'estompent lorsqu'une contribution publique est prélevée pour décourager les activités taxées ${ }^{98}$. En cas de non-respect d'un acte concerté non obligatoire en droit international public ${ }^{99}$, l'entrave à des relations économiques ou commerciales normales peut constituer une mesure privilégiée de rétorsion ${ }^{100}$. Ce type de mesure suscite bien évidemment peur, angoisse et anxiété.

La pression peut également être de nature réglementaire, décisionnelle ou juridictionnelle. Le destinataire obéira dans ce cas par crainte de voir mise en œuvre par les autorités la menace d'adopter une réglementation, de rendre une décision ou de déposer un recours. L'effet est d'autant plus fort que la crédibilité de l'exécution de la menace est imminente.

D'un côté du spectre, cette pression peut être très diffuse, de nature politique, à l'instar des arrangements non obligatoires et non formalisés visant à éviter un acte réglementaire. Dans ce type d'acte, la prestation étatique déterminante consiste en l'engagement de l'Etat à renoncer à adopter un acte réglementaire à condition que les particuliers adoptent une autoréglementation dont le contenu sera conçu pour éviter d'adopter l'acte juridique, formant un substitut plus favo-

\footnotetext{
94 Timsit, Gérard, «Sur l'engendrement du droit», Revue de droit public 1988, p. 49.

${ }^{95}$ Flückiger 2004 [note 11], p. 249s.

${ }^{96}$ Hart, H.L.A., Le concept de droit, Bruxelles 1976, p. 58.

${ }^{97}$ Ost/Kerchove 2002 [note 3], p. 221ss.

${ }^{98}$ Hart, H.L.A., Le concept de droit, Bruxelles 1976, p. 58; Ost/Kerchove 2002 [note 3], p. 232.

${ }^{99}$ Virally, Michel, «La distinction entre textes internationaux de portée juridique et textes internationaux dépourvus de portée juridique (à l'exception des textes émanant des organisations internationales): rapport provisoire», Annuaire de l'Institut de droit international, vol. 60, tome I, Paris

${ }^{100}$ Nguyen 2002 [note 71], ch. 573, p. 958.
} 1983 , p. 231 s. 
rable pour les particuliers ${ }^{101}$. On songe aux gentlemen's agreements ${ }^{102}$ auxquels la Banque nationale a eu recours dès $1930^{103}$ ou aux accords de branche ${ }^{104}$ en droit de l'environnement depuis les années $1970^{105}$. De l'autre côté du spectre, la pression peut être juridicisée, tout en restant de fait, lorsque l'on se trouve en présence d'une loi Damoclès. Ce type de loi institutionnalise la partie de l'accord liant l'Etat aux particuliers en garantissant juridiquement que la collectivité publique n'adoptera pas une réglementation étatique dans un domaine particulier en échange de l'adoption «volontaire» d'une régulation privée autonome ${ }^{106}$. Les particuliers ne sont pas juridiquement obligés d'adopter une solution auto-réglementée mais la peur que l'Etat édicte une réglementation, moins favorable, dicte leur comportement en fait. La doctrine n'est pas dupe et a pu écrire à propos de tels accords de «liberté conditionnelle accordée aux entreprises» ${ }^{107}$, de $\langle$ sanction» ${ }^{108}$, ou a comparé ce type d'acte non obligatoire à celui du bourreau présentant l'instrument du supplice comme premier degré de torture... ${ }^{109}$ C'est une démonstration de la manière dont la soumission peut se doubler d'un sentiment de liberté, menant à la situation paradoxale de «soumission forcée avec libre choix» ou de «soumission librement consentie», extrêmement efficace au demeurant comme les travaux en psychologie sociale l'ont montré ${ }^{110}$. On trouve des exemples en droit de l'environnement, en droit énergétique, en droit cinématographique ainsi qu'en droit de la consommation ${ }^{111}$.

La crainte de l'édiction d'une décision agit de manière analogue. La loi confère dans ce cas aux actes non obligatoires un effet appréciatif qualifié en instaurant la compétence de rendre unilatéralement une décision dans l'hypothèse où l'acte non obligatoire ne serait pas suivi ou rejeté par ses destinataires. La menace de décider agit derechef comme une épée de Damoclès ${ }^{112}$.

${ }^{101}$ Flückiger 2004 [note 11], p. 211ss (réf. cit.).

102 Poltier, Etienne, «Les gentlemen's agreements à participation publique », Revue de droit suisse I 1987, p. 395.

${ }^{103}$ Zufferey, Jean-Baptiste, La réglementation des systèmes sur les marchés financiers secondaires: contribution dogmatique et comparative à l'élaboration d'un droit suisse des marchés financiers, Fribourg 1994, p. 318.

${ }^{104}$ Pfenninger, Hanspeter, Rechtliche Aspekte des informellen Verwaltungshandelns, Fribourg, 1996, p. 25.

${ }^{105}$ Exemples in Flückiger 2004 [note 11], p. 213s.

${ }^{106}$ Flückiger, Alexandre, «La loi Damoclès», in Mélanges en l'honneur de Pierre Moor: théorie du droit, droit administratif, organisation du territoire, Berne 2005, p. 233ss.

${ }^{107}$ Matthey, Blaise, «La révision de la LPE dans la perspective de l'économie et des entreprises », Droit de l'environnement dans la pratique 1996, p. 588.

${ }^{108}$ Müller, Markus/ Müller-Graf, Thomas, «Staatliche Empfehlungen: Gedanken zu Rechtscharakter und Grundrechtsrelevanz», Revue de droit suisse II 1995,, p. 374.

${ }^{109}$ Kautz, Steffen, Absprachen im Verwaltungsrecht: Zulässigkeit, Grenzen und Folgen, Berlin 2002, p. 246 (réf. cit.).

${ }^{110}$ Voir ci-dessous ch. 3 .

${ }^{111}$ Flückiger, Alexandre, «La loi Damoclès », in Mélanges en l'honneur de Pierre Moor: théorie du droit, droit administratif, organisation du territoire, Berne 2005, p. 233ss; Flückiger 2004 [note $11]$, p. 216.

${ }^{112}$ Exemples in Flückiger 2004 [note 11], p. 218ss et 251ss. 
La peur d'un procès est également un instrument pour inciter les parties non seulement à conclure un arrangement non obligatoire, mais également à le respecter $^{113}$. L'efficacité est d'autant plus grande que les chances de succès sont élevées et que les instances redoutées sont longues et coûteuses. L'efficacité du droit de recours des organisations de protection de l'environnement repose sur ce mécanisme psychologique. L'efficacité de ce droit ne dépend en effet pas de son utilisation effective, qui est extrêmement faible, mais de la menace concrète que ce droit représente aux yeux des constructeurs, en raison de son utilisation judicieuse et couronnée de succès ${ }^{114}$.

Lorsqu'un tribunal juge, ou risque de juger, qu'une recommandation déterminée, publique ou privée, s'applique pour définir les limites de la négligence ou de l'imprudence, la crainte d'engager sa responsabilité pour actes illicites, voire licites, est un facteur puissant pour inciter un individu ou une autorité à respecter un acte non obligatoire. Les destinataires demeurent pourtant libres d'adopter un autre comportement et ne seront frappés qu'a posteriori, c'est-à-dire au moment de la survenance d'un dommage ${ }^{115}$.

\subsubsection{La honte}

Provoquer la honte et la culpabilité est monnaie courante en droit pénal. Dans le domaine de la soft law, elle déploiera similairement le plus d'effet en lien avec la peur de leur survenance. L'atteinte à la réputation d'une entreprise peut en effet coûter très cher à celle-ci; d'où la nécessité de maintenir l'image, de préserver une réputation irréprochable afin de prévenir la honte, l'embarras ou l'humiliation ${ }^{116}$. L'économie privée peut y parvenir par le biais d'instruments de soft law, à l'instar $\mathrm{du}$ Swiss Code of Best Practice for Corporate Governance ${ }^{117}$ ou de nombreux codes de déontologie, de bonne conduite ou d'éthique dont certaines professions se dotent (avocats, médecins, journalistes, etc.), y compris l'administration publique ${ }^{118}$.

${ }^{113}$ Müller-Graf, Thomas, Entrechtlichung durch Informalisierung?: ein Beitrag zur Handlungsformen- und zur Rechtsverhältnislehre im Verwaltungsrecht, Bâle, etc. 2001, p. 174.

${ }^{114}$ Flückiger, Alexandre/Morand, Charles-Albert/Tanquerel, Thierry, Evaluation du droit de recours des organisations de protection de l'environnement, Berne, 2000, p. 85ss.

${ }^{115}$ Flückiger 2004 [note 11], p. 254. Sur la responsabilité de l'Etat pour ses activités non décisionnelles, voir Schwarzenbach, Hans Rudolf, Staatshaftung bei verfügungsfreiem Verwaltungshandeln: mit praktischer Anleitung zum Vermeiden/Vermindern dieser wachsenden Risiken (Riskmanagement), Berne 2006.

${ }^{116}$ Roth, Monika, Soft law: Ordnungsvisionen in flux: eine Standortbestimmung mit Fokus auf compliancerelevante Fragestellungen, Berne 2006, p. 103ss.

${ }^{117}$ Adopté le 25 mars 2002 par l'organisation faîtière economiesuisse fixant une ligne de conduite et formulant des recommandations (Forstmoser, Peter, "Corporate governance in der Schweiz: besser als ihr Ruf», Corporate governance: Symposium zum 80. Geburtstag von Arthur Meier-Hayoz, Zurich 2002, p. 45ss et 63s; Crone, Hans Caspar von der, «Freiheit und Verantwortung in der Corporate governance», Corporate governance: Symposium zum 80. Geburtstag von Arthur Meier-Hayoz Zürich, Zurich 2002, p. 73). Sur les codes de conduite des entreprises multinationales, voir Roth, Monika, Soft law: Ordnungsvisionen in flux: eine Standortbestimmung mit Fokus auf compliance relevante Fragestellungen, Berne 2006, p. 13ss.

${ }^{118}$ Schindler, Benjamin, «Ethikförderung in der Verwaltung: Modetrend oder Notwendigkeit?», Schweizerisches Zentralblatt für Staats- und Verwaltungsrecht 2003, p. 61ss. 
Les procédés dits de naming and shaming consistant à dénoncer publiquement des individus, des organisations ou des Etats ${ }^{119}$ afin de provoquer l'indignation, la colère, la rage ou le dégoût misent sur ces mêmes ressorts. L'effet de tels procédés concernant des individus peut s'avérer bien plus douloureux que le recours à des actes juridiques obligatoires comme l'exemple de la mise en garde contre les produits dangereux le montre: la nécessaire publicité atteint de toute évidence plus le producteur que la simple notification d'une interdiction de distribution, en raison de l'atteinte à son image ${ }^{120}$. L'atteinte au droit fondamental à la protection de la sphère privée qui en résulte (art. 13 de la Constitution fédérale suisse [ciaprès: Cst.]) a pour conséquence que de tels procédés ne pourront être justifiés que s'ils reposent pour le moins sur une base légale (art. $36 \mathrm{al} .1^{\mathrm{er}} \mathrm{Cst}$.), exigence contribuant à leur juridicisation. Leur efficacité est discutée s'agissant des acteurs étatiques pour le moins, à l'instar de la mise en œuvre de la méthode ouverte de coordination en droit européen (mode non contraignant de coordination de politiques publiques) ${ }^{121}$.

Les campagnes d'informations misant sur la peur d'une maladie ou d'un accident ont souvent pour corollaire de provoquer honte ou culpabilité auprès de leurs destinataires. Tel est le cas dans la promotion du don d'organe où, implicitement ou explicitement, le non-donateur ne peut que se sentir coupable de ne pas vouloir sauver la vie des malades victimes de la pénurie d'organes et en éprouver de la honte ou de l'embarras ${ }^{122}$. Il en va de même dans la lutte contre le littering ${ }^{123}$, c'est-à-dire le fait de joncher la voie publique de déchets de toutes sortes: le malpropre doit se sentir coupable et devrait avoir honte de faire nettoyer ses immondices par autrui. La campagne stop-sida de l'Office fédéral de la santé publique représentant un père de famille assis avec son épouse et sa petite fille

${ }^{119}$ Stein, Jana von, «'Naming and Shaming' in the United Nations Commission on Human Rights: When and How Does it Matter?» Paper presented at the annual meeting of the International Studies Association 48th Annual Convention (publié sur www.allacademic.com/meta/ p179882_index.html). On retrouve le même procédé à l'oeuvre pour la mise en oeuvre de la méthode ouverte de coordination (MOC) en droit européen (Collignon, Stefan et al., «La Stratégie de Lisbonne et la méthode ouverte de coordination: 12 recommandations pour une stratégie à plusieurs niveaux plus efficace», Policy paper no 12, 2006, p. 10 [www.notre-europe.eu - rubrique travaux]).

${ }^{120}$ Kautz, Steffen, Absprachen im Verwaltungsrecht: Zulässigkeit, Grenzen und Folgen, Berlin 2002, p. 86, avec réf. cit. à la doctrine allemande. Voir également ibidem, p. 156 et 281s; Tschannen, Pierre, «Amtliche Warnungen und Empfehlungen», Revue de droit suisse II 1999, p. 432; Nützi, Patrick, Rechtsfragen verhaltenslenkender staatlicher Information: Strukturen, Zulässigkeit, Haftung : illustriert an den Beispielen AIDS und Listeriose, Berne 1995, p. 181ss

${ }^{121}$ La mise en œuvre de la méthode ouverte de coordination en droit européen repose sur un système de pression par les pairs qui «n'a toutefois pas correctement fonctionné, en partie du fait que les membres ne souhaitent pas «nommer et mortifier» leurs pairs. Les acteurs sont peu motivés pour engager ce type de procédure: les différents membres craignent de se faire des ennemis et d'en payer les conséquences négatives dans d'autres domaines politiques où ils ont peu progressé.» (Collignon 2006 [note 119], p. 10).

${ }^{122}$ Hammer, Raphaël, «Le traitement médiatique de la pénurie et du don d'organes: variations discursives et normatives dans la presse francophone suisse», contribution à un ouvrage collectif (Thiel, M.-J., éd.), Presses Universitaires de Strasbourg (à paraître).

${ }^{123}$ Flückiger, Alexandre, «Le littering ou la mode des mots en vogue contre la vague des déchets sauvages », UmweltUmfeldUmbruch: Streiflichter für Monika Kölz, Zurich, septembre 2007, p. 25s. 
autour d'un gâteau d'anniversaire avec en arrière-plan assombri ses anciennes amantes affriolantes ${ }^{124}$ est conçu pour provoquer sa culpabilité et sa honte envers sa charmante famille qui lui fait aveuglément confiance. S'il devait stigmatiser certains groupes de la population, le recours à de telles émotions contreviendrait à l'interdiction de la discrimination, en particulier au regard du critère du mode de vie (art. 8 al. 2 Cst.).

\subsubsection{La joie}

La joie ainsi que toutes les émotions positives qui peuvent en découler telles que la fierté, l'estime de soi, le désir ou le plaisir sont des émotions permettant de modifier le comportement des individus sans les forcer.

Tel est le sens de tous les instruments économiques redistributifs qui jouent sur le plaisir à gagner de l'argent et le désir du profit. Ainsi lorsqu'une recommandation est doublée d'un subventionnement étatique, l'incitation économique renforce le caractère appréciatif de la recommandation. Bien que le respect du comportement subventionné demeure à la libre appréciation des destinataires, la probabilité de modification volontaire du comportement augmente en fonction de la somme en jeu. Lorsque celle-ci est importante, elle confère à la recommandation sous-jacente un effet appréciatif pouvant aller jusqu'à un effet de fait contraignant. Tel est le cas du paysan dont le salaire dépend des subventions étatiques: sa libre volonté de ne pas cultiver ses céréales de manière extensive est d'autant plus limitée que les moyens d'en faire vivre sa famille en dépend: s'il poursuit une culture intensive, il n'aura pas droit aux paiements directs correspondants. Cette analyse ne vaut évidemment que pour les subventions promises visant un comportement non obligatoire; elle ne vaut pas pour le soutien financier de l'Etat à des actes obligatoires et ne concerne pas non plus le rapport entre les autorités et la personne subventionnée dès le versement de la subvention dans la mesure où un lien obligatoire découle de la décision ou du contrat de subventionnement ${ }^{125}$.

La remise de prix ou de distinctions par les autorités aux personnes les plus méritantes dont on récompense l'attitude à suivre est une recommandation indirecte d'adopter un comportement déterminé. Ce type de recommandation parie sur la joie, la fierté, le plaisir du récipiendaire, voire le désir, l'envie ou la jalousie qu'une telle récompense pourrait susciter chez les autres. Entre autres exemples, on évoquera les prix ou d'autres distinctions dans le domaine cinématographique (art. 7 LCin ${ }^{126}$; ordonnance sur le Prix du cinéma suisse ${ }^{127}$ ) afin d'encourager la création dans ce secteur.

Les campagnes d'information peuvent également faire vibrer la corde de la joie et du bonheur: le plaisir de manger sainement pour lutter contre l'obésité; le bonheur de voir un jeune athlète greffé du cœur pour promouvoir le don d'organe; la magie d'une promenade dans des sous-bois verdoyants pour prévenir la mort des forêts. En outre, le don d'organe peut être gratifié d'un geste symbolique de

\footnotetext{
${ }^{124}$ Campagne 2007 (www.bag.admin.ch/hiv_aids).

${ }^{125}$ Flückiger 2004 [note 11], p. 250s.

${ }^{126}$ RS 443.1 .

${ }^{127}$ RS 443.116 .
} 
remerciement après la transplantation (art. 6 al. 2, let. c de la loi fédérale sur la transplantation ${ }^{128}$ ). La campagne fédérale de lutte contre l'alcoolémie mise spécifiquement sur l'estime de soi et la fierté à retrouver («regarde-toi en face plutôt qu'au fond d'un verre ») ${ }^{129}$. La nouvelle campagne fédérale de lutte contre le tabagisme consiste à féliciter les personnes et les organisations ayant joué un rôle pionnier dans la promotion d'un environnement sans fumée afin de faire un maximum d'émules ${ }^{130}$.

Enfin, on peut revenir sur le phénomène de conformisme social que nous avons évoqué au chapitre de la peur. Le conformisme de complaisance par lequel l'individu craignant d'assumer un conflit avec le groupe préfère le plaisir de la tranquillité doit être distingué du conformisme d'identification. Ce dernier ne repose pas sur la peur mais sur la joie, la fierté ou le désir de reconnaissance lorsque l'individu se conforme pour s'établir dans le groupe, conserver des relations positives avec ce dernier dans l'espoir d'obtenir éventuellement prestige et visibilité ${ }^{131}$.

Enfin on notera que le plaisir s'avérera souvent être un facteur s'opposant à la mise en œuvre des règles de soft law. L'individu est constamment amené à mettre en balance les peurs que lui inspire une campagne anti-tabac ou antisida avec le plaisir de fumer ou de s'accoupler sans préservatif.

\subsubsection{La surprise}

La surprise est l'une des six émotions de bases de Paul Ekman au même titre que la colère, la peur, le dégoût, la joie et la tristesse ${ }^{132}$.

La surprise est omniprésente dans la hard law, mais s'y inscrit en négatif dans la mesure où le système juridique est fondamentalement bâti sur le principe de la sécurité du droit, même si la prévisibilité absolue de la règle de droit n'est depuis longtemps plus qu'une illusion en raison de la texture fondamentalement ouverte des règles de droit ${ }^{133}$, laissant un espace à la créativité de la jurisprudence et donc à la surprise. Celle par exemple de voir un jour la protection de l'environnement garantie par la Cour européenne des droits de l'homme à Strasbourg sur le fondement d'une disposition garantissant le droit au respect de la vie privée et familiale $(\text { art. } 8 \mathrm{CEDH})^{134}$ ou la surprise plus coutumière devant l'annonce du résultat d'un

${ }^{128}$ RS 810.21.

${ }^{129}$ www.bag.admin.ch/themen/drogen/00039/00602

${ }^{130}$ www.bag.admin.ch/themen/drogen/00041/00617

${ }^{131}$ Baggio, Stéphanie, Psychologie sociale, Bruxelles 2006, p. 22; Doise, W., Deschamps, J.-C. et Mugny, G., Psychologie sociale expérimentale, 2e éd., Paris, A. Colin, 1991, chapitre 7.

${ }^{132}$ Ekman, Paul, «An Argument for Basic Emotions», Cognition and Emotion, 1992, 6, 169ss; Ekman, Paul, Emotions in the Human Face, Cambridge 1982.

${ }^{133}$ Moor, Pierre, Pour une théorie micropolitique du droit, Paris 2005, p. 56ss; Papaux, Alain, Introduction à la philosophie du «droit en situation»: de la codification légaliste au droit prudentiel, Bruxelles [etc.] 2006, p. 192ss.

${ }^{134}$ L'article 8 s'applique en effet depuis une quinzaine d'années aux atteintes graves à l'environnement qui peuvent affecter le bien-être d'une personne et la priver de la jouissance de son domicile de manière à nuire à sa vie privée et familiale (CourEDH, López Ostra c. Espagne, 9 décembre 1994) La requérante se plaignait en l'espèce des émanations de gaz, des odeurs pestilentielles et des contaminations émanant d'une station d'épuration située à quelques mètres de son domicile à Lorca. 
recours. Lorsque la jurisprudence est bien établie dans un domaine, le juge est en principe obligé d'en annoncer à l'avance un éventuel changement ${ }^{135}$, précisément afin d'atténuer l'effet de surprise. Dans le domaine des droits politiques, le Tribunal fédéral vient de reconnaître que la surprise était dans la nature même de cette institution emblématique de la démocratie directe helvétique réunissant tous les citoyens en une assemblée solennelle sur une place publique du chef-lieu: la Landsgemeinde ${ }^{136}$.

La soft law présente une caractéristique intéressante à ce propos en rapport avec la hard law en ce sens que la première a souvent une fonction expérimentale permettant de tester une future règle de droit potentielle (droit «vert» qui ne demande qu'à mûrir $)^{137}$. L'Etat peut ainsi étudier la réaction des destinataires et retirer les mesures testées ou les pérenniser en fonction de l'impact observé ${ }^{138}$. La soft law habitue ainsi les citoyens, les acclimate progressivement à un nouveau régime en les prévenant de toute surprise le jour de la maturation en hard law (la fumée est d'abord déconseillée, puis interdite dans les lieux publics; le port du casque et de la ceinture de sécurité est d'abord préconisé puis imposé; etc.). On retrouve une variante de ce processus lorsqu'une planification non impérative crée au fur et à mesure des faits accomplis qu'il devient de plus en plus difficile de contrer pratiquement au fil du temps ${ }^{139}$. La soft law permet de créer une accoutumance graduelle déjouant toute surprise ultérieure et la réaction possible de rejet susceptible de l'accompagner.

En matière de don d'organe, le Comité d'éthique français a admis sous certaines conditions la technique de l'approche anticipée - c'est-à-dire l'approche avant le décès - des proches d'un sujet en coma grave en vue d'un prélèvement. Le but est de mieux préparer les proches au décès et à l'éventualité du prélèvement afin de réduire le taux de refus ${ }^{140}$. Il s'agit d'habituer progressivement et de ne pas provoquer un effet de surprise trop grand au moment clé. De manière plus générale, les équipes médicales de neuroréanimation commencent à s'adjoindre des psychologues en instaurant des protocoles d'accueil des familles permettant le passage en douceur du «deuil au don», atténuant l'effet de surprise ${ }^{141}$. Afin de

${ }^{135}$ Probst, Thomas, Die Änderung der Rechtsprechung, Bâle, etc. 1993 ; Moor, Pierre, Droit administratif: les fondements généraux, vol. I, $2^{\mathrm{e}}$ éd., Berne 1994, p. 73ss.

${ }^{136}$ Arrêt du Tribunal fédéral suisse (ci-après: ATF) 132 I 291, 295 (www.bger.ch).

${ }^{137}$ Voir Abi-Saab, Georges, «Cours général de droit international public », Recueil des cours de l'Académie de droit international, t. 207, 1997, p. 207,210; Morand 1999 [note 3], p. 170. On notera un phénomène analogue en hard law: l'expérimentation législative (Flückiger, Alexandre, «Voter, élire et signer par Internet: le droit expérimental à l'épreuve de la sécurité », in Journées 2002 d'informatique juridique, Berne 2003, p. 107ss [réf. cit.]). En droit allemand, voir Maass, Volker, Experimentierklauseln für die Verwaltung und ihre verfassungsrechtlichen Grenzen : zugleich ein Beitrag zu $\S 7$ a BerlHG, Berlin 2001).

${ }^{138}$ Flückiger 2004 [note 11] (réf. cit.)

${ }^{139}$ Sur de tels effets en matière de planification, voir Flückiger, Alexandre, Le régime juridique des plans: l'exemple du plan de gestion des déchets, Berne, 1996, p. 117ss (réf. cit.).

${ }^{140}$ Comité d'éthique, séance du 10 janvier 2005, in Agence de la biomédecine, Recommandations du Comité d'Ethique de l'Etablissement français des Greffes 1997-2005, Paris 2005 (http://www. agence-biomedecine.fr/fr/experts/doc/saisines-ce-efg.pdf).

${ }^{141}$ Novartis, La lettre de la proximologie, no 36, janvier 2007, p. 5 (www.proximologie.com). 
rendre le public attentif à la pénurie d'organes, une télévision hollandaise a quant à elle utilisé l'effet de surprise de manière très douteuse dans une émission où les téléspectateurs apprenaient à la fin que la soi-disant donneuse de rein était une comédienne ${ }^{142}$.

Enfin, certaines techniques de manipulation jouent sciemment sur l'effet de surprise comme celle de la porte-au-nez ou du leurre ${ }^{143}$.

\subsubsection{L'empathie}

L'empathie est une notion désignant le mécanisme psychologique par lequel un individu peut comprendre les sentiments et les émotions d'une autre personne, sans les ressentir lui-même ${ }^{144}$. Il s'agit plus précisément d'une faculté de ressentir les émotions en général plutôt qu'une émotion en soi.

L'empathie est certainement une qualité indispensable au succès des procédures non juridiques de règlement des différends telles que la médiation ${ }^{145}$. Elle est également à l'œuvre, avec la compassion, la sympathie ou la pitié, dans le recours aux récits biographiques qui facilitent l'expression émotionnelle en faisant vibrer le lecteur, l'incitant de la sorte à agir dans un sens déterminé. Telle est la technique couramment utilisée dans les campagnes de promotion du don d'organe où les témoignages des transplantés insufflent une dimension humaine et palpable dans la souffrance d'individus en attente d'une greffe, donnant d'un coup un visage émotionnel à la pénurie causée par le nombre insuffisant de donneurs ${ }^{146}$ . Au niveau de la relation entre le personnel médical et la famille du donneur, le taux de consentement est plus élevé chaque fois que les médecins font preuve d'empathie alors qu'il diminue en cas de froideur ${ }^{147}$. Aussi n'est-il dès lors pas étonnant que les directives médico-éthiques de l'Association suisse des sciences médicales sur le diagnostic de la mort dans le contexte de la transplantation d'organes du 24 mai $2005^{148}$ précisent que «l'information sur la défaillance du cerveau après lésion cérébrale primaire [...] devra se faire dans le calme et avec empathie. Ensuite - et au terme d'un temps adéquat - on peut évoquer avec les proches la question d'un éventuel don d'organes ${ }^{149}$.

${ }^{142}$ www.nzz.ch/2007/06/01/vm/newzzF2F39I3L-12.html

${ }^{143}$ Voir ci-dessous ch. 3.1.

${ }^{144}$ Davis, M. H., «Empathy», Stets, J. E./ Turner, J. H. (éd.), Handbook of the sociology of emotions, New York 2006, p. 443ss; Deonna, Julien, «The structure of Empathy », Journal of Moral Philosophy 4.1, Avril 2007.

${ }^{145}$ Guy-Ecabert, Christine, Procédure administrative et médiation: inscription d'un modèle procédural dans un contexte en mutation, Zurich etc. 2002, p. 255. Sur le rôle, controversé, que devrait jouer l'empathie auprès du juge, voir ci-dessus en note de bas de page 34 .

${ }^{146}$ Hammer, Raphaël, «Le traitement médiatique de la pénurie et du don d'organes: variations discursives et normatives dans la presse francophone suisse», contribution à un ouvrage collectif (Thiel, M.-J., dir.), Presses Universitaires de Strasbourg (à paraître).

${ }^{147}$ Voir l'étude d'Annemarie Kesselring, cité in «L'art de parler du don d'organes avec les proches », Fonds National suisse - Horizons, juin 2005, p. 19s.

148 www.samw.ch

${ }^{149}$ Ch. 4 des directives. 
Cette émotion peut également être liée à la générosité, la gratitude, la bonté, l'altruisme, la solidarité et le dévouement; éléments présents à un titre ou à un autre dans toute campagne encourageant le don d'organe ${ }^{150}$. Dans le cadre des campagnes de lutte contre le sida, l'exigence de solidarité est ainsi expressément défendue par l'Office fédéral de la santé publique afin de ne pas stigmatiser et discriminer certains modes de vie et pratiques ${ }^{151}$.

\section{LES TECHNIQUES DE MANIPULATION}

$\mathrm{Au}$ lieu de sensibiliser la population en sollicitant des arguments de nature émotionnelle pour lui faire changer de comportement sans l'aide de règles de droit, les psychologues sociaux ont démontré que certaines techniques permettaient de manipuler les individus à leur insu afin de leur faire faire librement des actes qu'ils n'auraient pas accomplis sans ces manipulations.

On songe aux théories de l'engagement (ch. 3.1), à l'utilisation stratégique du phénomène de la dissonance cognitive (ch. 3.2) et aux effets de contexte (contraste et compromis) (ch. 3.3).

\subsection{Les théories de l'engagement}

Les théories de l'engagement (commitment), dont l'efficacité semble selon certains supérieure aux stratégies de sensibilisation et d'information, mais qui peuvent être utilisées en complément ${ }^{152}$, ont été popularisées dans le monde francophone par deux auteurs qui les ont regroupées dans un livre appelé «Petit traité de manipulation à l'usage des honnêtes gens» ${ }^{153}$. Interrogés sur la dimension éthique de telles techniques permettant d'obtenir une «soumission librement consentie ${ }^{154}$, ces auteurs répondent que «les pratiques d'engagement peuvent scandaliser celles et ceux qui sont intimement convaincus que toute l'activité humaine est réductible à une activité de libre décision, éclairée par un ensemble d'arguments que le décideur aurait accepté de recevoir, qu'il aurait traité dans la plénitude de son intelligence, ou qu'il aurait élaboré en lui-même en l'absence de toute influence [...] Près d'un siècle de psychologie scientifique nous conduit à refuser cette trop belle image de l'activité humaine et à dénoncer les effets pervers de sa promotion idéologique. Aussi, toute discussion éthique doit admettre comme préalable que ce n'est pas parce qu'on s'agrippe à cette image qu'on est un ange,

${ }^{150}$ Voir Hammer (cit. ci-dessus note 122).

${ }^{151}$ www.bag.admin.ch/hiv_aids/00828

${ }^{152}$ Voir l'efficacité comparée des stratégies de sensibilisation, de persuasion et d'engagement dans le cadre de la lutte contre le sida en rapport avec le port du préservatif (Joule, Robert-Vincent; Beauvois, Jean-Léon, La soumission librement consentie: comment amener les gens à faire librement ce qu'ils doivent faire? $5^{\mathrm{e}}$ éd., Paris 2006, p. 137ss).

${ }^{153}$ Joule, Robert-Vincent; Beauvois, Jean-Léon, Petit traité de manipulation à l'usage des honnêtes gens, Grenoble 2002. Voir ég. Fabrice d'Almeida, La manipulation, $2^{\mathrm{e}}$ éd., Paris 2005, p. $58 \mathrm{ss}$.

${ }^{154}$ Joule/Beauvois 2006 [note 152] pour des applications en matière d'économie d'énergie, de prévention des accidents de travail et de lutte contre le sida. 
ni parce qu'on y renonce qu'on est un démon.» ${ }^{155}$ Appliquée à l'Etat de droit, la question éthique devient juridique; elle pourrait se résumer à celle de savoir dans quelle mesure il serait admissible pour un Etat de suivre les éventuels préceptes d'un Petit traité de manipulation à l'usage des gouvernements honnêtes dans le but d'améliorer la mise en œuvre de leurs politiques publiques.

De telles pratiques ne recourent pas directement aux émotions individuelles mais les conditionnent de manière inconsciente, en particulier par la tromperie, le leurre ou la surprise. Contrairement à la hard law, et de même que la soft law, le sujet est placé dans une situation qui lui confère un sentiment de libre choix. Cette perception de liberté est déterminante pour le succès des techniques manipulatoires. L'individu doit avoir l'impression d'une décision libre et éclairée, dégagée de toute influence, bien qu'il se conduise d'une manière différente de ce qu'aurait été la sienne s'il n'avait pas été manipulé à son insu. Les théories de l'engagement sont à la base de tels procédés ${ }^{156}$. Le principe est que la conduite du sujet est déterminée par une ou plusieurs conduites préalables préparées à son insu. Les sujets sont pris dans un engrenage duquel ils peinent d'autant plus à sortir que le temps avance $^{157}$. Plusieurs méthodes peuvent être utilisées:

- Le pied-dans-la-porte qui consiste à faire précéder la demande principale d'une demande anodine, peu coûteuse et sans rapport nécessaire avec la requête principale (par exemple demander l'heure) avant de solliciter le sujet pour lui demander d'accomplir l'acte voulu qu'il aurait probablement refusé de faire si la requête n'avait pas été précédée de cette demande préalable ${ }^{158}$.

- La porte-au-nez qui consiste au contraire à faire précéder la requête principale d'une demande excessive mais non abusive afin d'obtenir moins; le «moins » correspondant en réalité à ce que le demandeur veut exactement. L'effet de surprise apaisante crée chez le sujet une illusion de gain le rendant statistiquement plus apte à consentir ${ }^{159}$.

- L'amorçage qui consiste à obtenir d'un individu l'assentiment de réaliser une action dont on lui cache dans un premier temps le coût réel avant de l'informer ultérieurement complètement ${ }^{160}$.

- Le leurre, variante de l'amorçage, qui conduit le sujet à accomplir un comportement, attractif, dont le demandeur sait que son exécution sera impossible, avant que celui-ci l'en informe dans un second temps et propose au sujet une solution alternative, plus coûteuse, mais que le demandeur avait initialement en tête ${ }^{161}$.

\footnotetext{
155 Joule/Beauvois 2006 [note 152], p. 207.

${ }^{156}$ C.A. Kiesler, The Psychology of Commitment, New York, 1971

${ }^{157}$ Baggio, Stéphanie, Psychologie sociale, Bruxelles 2006, p. 28.

${ }^{158}$ Joule/Beauvois 2002 [note 153], p. 97ss.

159 Joule/Beauvois 2002 [note 153], p. 117ss

${ }^{160}$ Joule/Beauvois 2002 [note 153], p. 53ss.

${ }^{161}$ Joule/Beauvois 2002 [note 153], p. 68ss.
} 
Les conditions suivantes permettent d'obtenir l'engagement d'un individu dans un acte: «le déclarer libre; mettre en relief les conséquences de son acte; choisir un acte de coût élevé (rechercher le coût maximum de l'acte qui sera accepté); rendre l'acte le plus visible possible: lui donner un caractère public; souligner le caractère explicite de sa signification; faire en sorte que tout retour en arrière soit impossible; ne pas hésiter à faire réaliser cet acte plusieurs fois (ou s'il s'agit de décision, à faire réitérer la décision prise); éviter toute justification d'ordre externe (pas plus de promesse de récompense que de menace de punition); avancer une explication interne.» ${ }^{162}$

On soulignera en particulier la nécessité pour l'individu de se croire libre pour que l'effet escompté puisse être atteint. Les recherches empiriques en psychologie sociale ont démontré que le fait de dire explicitement à une personne qu'elle est libre de choisir après une proposition qu'on lui a faite augmente sensiblement le taux d'acceptation; le «sentiment de liberté» devenant même «une élégante technique de manipulation» en $\mathrm{soi}^{163}$. La soft law s'appuie précisément sur ce type de ressort en tant précisément qu'elle se définit comme n'étant pas juridiquement obligatoire. On se reportera également au concept de réactance psychologique par lequel les individus, pensant voir leur liberté comportementale réduite, parviennent à résister aux tentatives de persuasion ${ }^{164}$.

Ces méthodes diffèrent des techniques de persuasion examinées auparavant (ch. 2) en ce sens que les attitudes originellement voulues deviennent une conséquence du comportement et non l'inverse.

Ces techniques sont susceptibles de s'appliquer pour renforcer les campagnes d'information et de persuasion. Elles ont notamment été testées, avec succès, en matière d'économie d'énergie pour demander d'éteindre les lumières lorsque l'éclairage devient inutile et de baisser le chauffage plutôt que d'ouvrir les fenêtres lorsque l'on a chaud ${ }^{165}$, de prévention des accidents de travail pour demander à des ouvriers de porter des protection auditives dans un environnement bruyant ${ }^{166}$ et de lutte contre le sida pour utiliser des préservatifs ${ }^{167}$ et pour se soumettre à un dépistage ${ }^{168}$.

Une étude approfondie des stratégies de promotion du don d'organe a montré l'efficacité potentielle de recourir à de telles techniques. Le don du sang a été utilisé comme pied-dans-la-porte prédisposant à signer une carte de donneur d'organes avec les résultats suivants: les donneurs de sang sont tendanciellement plus nombreux à signer une carte de donneur d'organes que les non-donneurs (47\% versus $25 \%$ ); les donneurs de sang sont tendanciellement plus nombreux à signer une carte de donneur d'organes lorsqu'ils sont sollicités dans un centre de don de sang que dans un parc (55\% versus $35 \%)$; les donneurs de sang sollicités dans un

\footnotetext{
162 Joule/Beauvois 2006 [note 152], p. 72.

${ }^{163}$ Joule/Beauvois 2002 [note 153], p. 160.

${ }^{164}$ Baggio [note 131], p. 66ss.

165 Joule/Beauvois 2006 [note 152], p. 95ss.

166 Joule/Beauvois 2006 [note 152], p. 110ss.

${ }^{167}$ Joule/Beauvois 2006 [note 152], p. 127ss.

${ }^{168}$ Joule/Beauvois 2006 [note 152], p. 142ss.
} 
centre de don de sang sont significativement plus nombreux que les non-donneurs à signer une carte de donneur d'organes $(55 \% \text { versus } 25 \%)^{169}$. Non seulement la relation de l'Etat avec le grand public mérite d'être examinée mais également celle qui lie personnel médical avec les donneurs ou plutôt leur famille (approche anticipée des proches d'un comateux profond ${ }^{170}$; proposition de signer une carte de donneur après avoir refusé d'être un donneur vivant comme porte-au-nez).

Le recours à certaines de ces techniques par les autorités est hautement discutable, en particulier celles qui tablent sur la tromperie et le leurre dans la mesure où elles risquent de s'avérer contraires au principe constitutionnel selon lequel «les organes de l'Etat et les particuliers doivent agir de manière conforme aux règles de la bonne foi » ( $\operatorname{art} .5 \mathrm{al} .3 \mathrm{Cst}$. et art. $9 \mathrm{Cst}$.) et de la proportionnalité (art. 5 al. 2 Cst.) en ce qui concerne la technique de la porte-au-nez. Ce constat est d'autant plus évident dans les domaines où l'ordre juridique impose un consentement libre, éclairé et en toute connaissance de cause à la prise de décision, comme dans l'exemple du don d'organe (art. 8, 12 et 61 de la loi fédérale sur la transplantation $)^{171}$, domaine dans lequel l'Etat aurait désormais même, selon le Conseil fédéral s'exprimant sur le projet de loi, l'interdiction de diffuser des campagnes d'information visant à «accroître la propension au don d'organe» ${ }^{172}$.

\subsection{L'utilisation stratégique du phénomène de la dissonance cognitive (effet de l'obligation de motiver)}

Selon la théorie de la dissonance cognitive, l'individu éprouve un inconfort lorsqu'il est confronté à des « cognitions » incompatibles entre elles, entendant par cognition les connaissances qu'une personne a d'elle-même, de son comportement et de son environnement ${ }^{173}$. L'état de tension désagréable ainsi éprouvé motive sa réduction afin d'atteindre un équilibre cognitif (une consonance).

\footnotetext{
${ }^{169}$ Eyssartier, Chloé, Du don de sang au don d'organes: une recherche appliquée, Université d'Aix-Marseille (http://slhs.univ-fcomte.fr/rech/psycho/colloque/social/resumes.htm\#e).

${ }^{170}$ Voir ci-dessus note de bas de page 140.

${ }^{171}$ RS 810.21.

${ }^{172}$ Feuille fédérale 2002, p. 175, souhaitant toutefois que l'information diffusée incite le public «à se poser la question du don d'organes» (ibidem). La loi doit certes «contribuer à ce que des organes, des tissus et des cellules humains soient disponibles à des fins de transplantation» (art. 1 al. 2 de la loi fédérale sur la transplantation). La marge du législateur est cependant ténue dans la mesure où le Parlement a refusé de compléter l'article constitutionnel sur la médecine de transplantation (art. 119a Cst.) par un alinéa supplémentaire donnant mandat à la Confédération d'encourager le don d'organes en informant la population sur sa nécessité, toutefois «sans faire de l'incitation» (Proposition Christiane Brunner, BO CE 1998 629). Un parlementaire, opposé à l'amendement, a cependant jugé que le projet d'article constitutionnel n'interdisait pas à la Confédération d'exercer une action dans ce domaine (Antoine Cottier, BO CE 1998 630). La parlementaire ayant proposé l'amendement l'a retiré à la suite de l'assurance, donnée par la conseillère fédérale responsable, que la loi tiendrait compte du faible degré de sensibilisation du public à cette question (BO CE 1998 631). L'article 61 al. 2 de la loi fédérale sur la transplantation assigne ainsi des buts très limités à l'information du public; celle-ci doit viser «notamment à [...] donner à chacun la possibilité d'exprimer sa volonté concernant le don d'organes, de tissus ou de cellules en toute connaissance de cause; [...] faire connaître la réglementation et la pratique, notamment à présenter les conditions de prélèvement, d'attribution et de transplantation d'organes, de tissus et de cellules en Suisse.»

${ }^{173}$ Festinger, Léon, A theory of cognitive dissonance, Evanston [etc.] 1957, p. 9.
} 
Certains modes de réduction de la dissonance cognitive ont pu être utilisés stratégiquement pour modifier le comportement des individus. Tel est le cas lorsque l'on demande à une personne de motiver une solution à laquelle elle n'adhère pas: dans ce cas, elle tempère sa position initiale, cherchant à harmoniser le comportement qu'elle a eu avec ses idées ${ }^{174}$. Une expérience par exemple consistait à sonder des étudiants en psychologie de première année sur leur opinion relative à un concours de sélection pour entrer en deuxième année, sachant que les étudiants sont majoritairement opposés à cette mesure. Dans un premier temps, ils devaient donner leur avis sur la réforme; dans un second, ils devaient dire ce qui, selon eux, était bénéfique dans la réforme proposée et lister quelques arguments en faveur de l'instauration d'un concours, puis donner à nouveau leur opinion étant entendu qu'ils pourraient ainsi confirmer leur réponse. Il ressort de cette expérience que la moitié des étudiants avait un avis plus favorable après ${ }^{175}$.

Cette technique de motivation de la décision pour modifier le comportement peut être utilisée par les soignants chargés de recueillir le consentement des proches d'un donneur potentiel en cas de mort encéphalique pour augmenter le taux d'acceptation comme le montre ce témoignage français ${ }^{176}$ : après avoir réuni les proches dans une pièce «aménagée pour humaniser l'annonce du décès», l'infirmière coordinatrice de prélèvement leur demande si, à leur connaissance, le défunt s'était clairement déterminé en matière de don d'organe. Si la famille répond «Non, un prélèvement est hors de question», la coordinatrice repose la question: "Mais le défunt lui-même avait-il exprimé son opposition formelle?'. Cette question évite à la famille de se sentir impliquée ou coupable en cas de refus. Si la coordinatrice sent chez le conjoint ou chez toute autre personne qui a pris la parole, une hésitation, elle personnalise alors sa question. 'Madame, (monsieur) je comprends votre hésitation, mais qu'est-ce qui motive votre refus?' 'Le dernier cas que j'ai vécu' explique [1'infirmière], 'était celui d'une femme dont le mari était juif pratiquant. Elle refusait, craignant que son mari ne puisse pas être enterré en Israël'. Il est en effet fréquent que l'obstacle soit religieux. Mais les coordinatrices connaissent parfaitement les pratiques funéraires des trois grandes religions monothéistes et savent qu'aucune d'elles ne s'oppose formellement au don d'organes. 'Alors, je l'ai rassurée sur l'absence d'obstacle au plan civil et religieux, et dès que son inquiétude a été calmée, j'ai obtenu l'accord de la famille'.» ${ }^{177}$

Lorsque la famille répond à la coordinatrice qu'elle ne connaît pas l'opinion du défunt, «l'indécision enlise alors la discussion.» ${ }^{178}$ Une technique mixte, mêlant motivation et pied-dans-la-porte, est alors utilisée: «Là aussi, les coordinatrices

${ }^{174}$ Baggio [note 131], p. 62ss.

${ }^{175} 9 \%$ un avis moins favorable et $41 \%$ avec un avis inchangé (Baggio [note 131], p. 63). Cette auteure relève toutefois que l'expérience aurait dû se dérouler dans un climat de plus grande liberté pour que l'on puisse vraiment parler de dissonance cognitive en l'espèce (p. 64).

${ }^{176}$ Plus précisément de se renseigner auprès des proches si le défunt s'était opposé au don d'organe puisque le droit français connaît le système du consentement présumé (art. L 1232-1 du Code de la santé publique) - la pratique dont il est ici question se déroulant à l'hôpital Cochin à Paris («Don d'organe: l'entourage familial au centre de la décision », La lettre de la proximologie, 2007, 36, p. 3ss [www.proximologie.com/a_professionnels/a03_ressources/a03_01_lettre/docs/LP36.pdf]).

${ }^{177}$ La lettre de la proximologie 2007 [note 176], p. 5s.

${ }^{178}$ La lettre de la proximologie 2007 [note 176], p. 6. 
doivent faire preuve de technique. Le mieux est de les faire parler du défunt. Celuici, bien logiquement, se retrouve paré de toutes les qualités. Tolérant, large d'esprit, généreux. - 'Il aurait donné son sang par exemple', demande l'infirmière. - 'Mais bien entendu, il aurait donné son sang', répond la famille. - 'Alors peut-être auraitil acquiescé à l'idée de faire don d'un organe?' - 'Ah, peut-être en effet...'» ${ }^{179}$

Lorsque l'on prend en compte les techniques psychologiques entrant en jeu dans de pareils entretiens, il est manifeste qu'un consentement ainsi obtenu n'est ni éclairé, ni libre ${ }^{180}$. D'un autre côté toutefois, la recherche en psychologie sociale nous montre les limites mêmes du concept de décision véritablement «libre ${ }^{181}$.

En tous les cas, de pareils protocoles d'entretien devraient faire au minimum l'objet de directives étatiques - et publiées officiellement - afin de clarifier les limites de la manipulation admissible de celle qui ne l'est pas. Le chapitre 4 des directives médico-éthiques de l'Association suisse des sciences médicales sur le diagnostic de la mort dans le contexte de la transplantation d'organes du 24 mai $2005^{182}$ intitulé «Information et assistance des proches et de l'équipe soignantes » est une première tentative, mais de loin insuffisante. Le Conseil fédéral a bien réglé une partie de la question, mais pour le donneur vivant, et non pour le cadavérique, en précisant les règles en matière d'information, notamment le droit du donneur vivant «de s'opposer au don sans avoir à motiver son refus» (art. 9 al. 2, lit. i de l'ordonnance sur la transplantation ${ }^{183}$ ). La réglementation par directive trouve toutefois ses limites dans le cadre d'une telle relation en raison de la nature extrêmement émotionnelle de celle-ci; de telles circonstances ne se prêtent en effet guère à une réflexion calme et pondérée. Il s'ensuit qu'il est préférable de favoriser la prise de décision en amont, par la signature de cartes de donneurs.

\subsection{Les effets de contexte (effets de contraste et de compromis)}

Les effets de contexte (effets de contraste et de compromis) induisent des biais dans le processus de décision. Leur utilisation à des fins de marketing s'est imposée dès l'origine ${ }^{184}$. Elle a été testée récemment dans le processus de décision juridique ${ }^{185}$.

${ }^{179}$ La lettre de la proximologie 2007 [note 176], p. 6.

${ }^{180}$ Sur les exigences relatives à l'information procurée aux patients par le corps médical et la responsabilité du médecin, voir Tribunal fédéral, arrêt 4P.265/2002 du 28 avril 2003, publié in Revue de droit administratif et fiscal 2003 I, p. 635; ATF 127 Ib 197; Winiger, Bénédict, «Causalité : «l'influence psychologique en droit suisse», Chappuis, Christine/ Winiger, Bénédict (éd.), Les causes du dommage, 2006, p. 45s; Manaï, Dominique, Les droits du patient face à la médecine contemporaine, Bâle [etc.] 1999, p. 120.

${ }^{181}$ Joule/Beauvois 2006 [note 152], p. 207 [le passage est cité à la note 155].

182 Www.samw.ch

${ }^{183}$ RS 810.211.

${ }^{184}$ Huber, J./ Payne, J.W./ Puto, C., «Adding asymmetrically dominated alternatives: Violations of regularity and the similarity hypothesis », Journal of Consumer Research, 1982, 9, p. 90ss; Simonson, «Choice Based on Reason »: The Case of Attraction and Compromise Effects », Journal of Consumer Research, 1989, 16, p. 158ss.

${ }^{185}$ Schweizer, Mark, «Kontrast- und Kompromisseffekt im Recht am Beispiel der lebenslänglichen Verwahrung», ZStrR 4/2005, p. 438ss; Kelman, M./ Rottenstreich, Y./ Tversky, A., «Contextdependence in legal decision making », in Sunstein, Cass, Behavioral law and economics, Cambridge [etc.] 2000, p. 61ss; 
L'effet de contraste a pour conséquence que la même option est évaluée plus positivement en présence de solutions alternatives similaires moins favorables qu'en l'absence de tels choix ${ }^{186}$. On retrouve cet effet en optique dans l'illusion d'Hermann Ebbinghaus: un disque de même diamètre est perçu comme étant plus petit lorsqu'il est entouré par de plus grands.
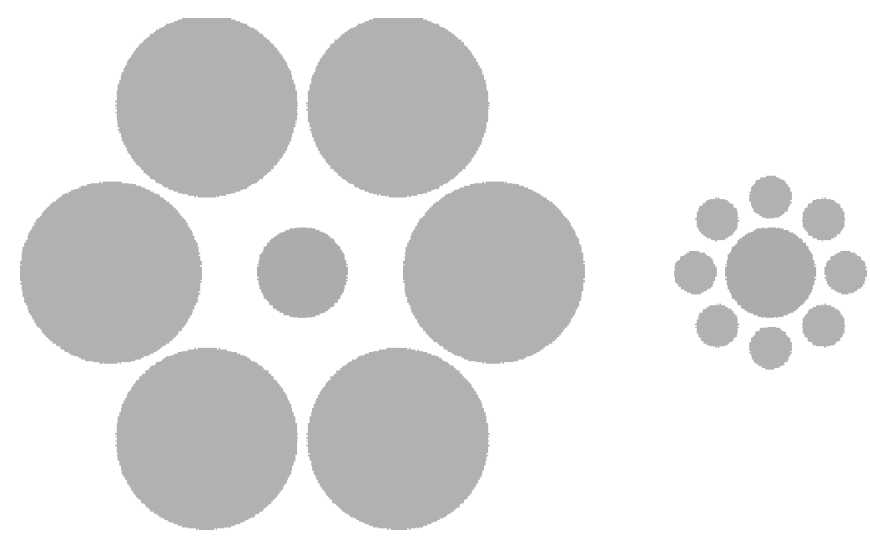

Un commerçant a pu ainsi augmenter la vente du même produit de $43 \%$ à $63 \%$ par rapport à un produit concurrent, moins cher mais de moindre qualité, simplement en en ajoutant un troisième de même qualité que le premier mais plus cher ${ }^{187}$. Cette expérience a été répliquée; elle est considérée comme robuste ${ }^{188}$.

L'effet de compromis résulte de l'observation selon laquelle la même option est évaluée plus favorablement lorsqu'elle est perçue comme étant intermédiaire dans un groupe de solutions alternatives que lorsqu'elle est présentée comme extrême ${ }^{189}$.

Une première expérience américaine a montré que, sur la base d'un même état de fait, la probabilité d'être condamné pour meurtre passait de $38 \%$ à $55 \%$ lorsque la qualification de meurtre qualifié était à disposition dans le choix des infractions $^{190}$. Cet effet de compromis a pour conséquence que le condamné risque une peine plus élevée lorsque la loi prévoit un état de fait qualifié, même s’il n'est pas condamné pour ce dernier chef ${ }^{191}$. L'expérience a été répliquée, avec des résultats analogues, auprès de 230 juges civils et pénaux des cantons de Bâle, de Berne et des Grisons en comparant leurs jugements sur la base d'un même état de fait selon que la possibilité de l'internement à vie (plus grave) était à leur disposition ou non dans la panoplie des sanctions pénales ${ }^{192}$. L'introduction d'une option extrême

\footnotetext{
${ }^{186}$ Kelman et al. 2000 [note 185], p. 61.

${ }^{187}$ Huber et al. 1982 [note 184], p. 95, 97.

${ }^{188}$ Schweizer 2005 [note 185], ch. III, lit. A.

${ }^{189}$ Kelman et al. 2000 [note 185], p. 61; Simonson 1989 [note 184].

${ }^{190}$ Kelman et al. 2000 [note 185], p. 67.

${ }^{191}$ Schweizer 2005 [note 185], ch. V, lit. A.

${ }^{192}$ Schweizer 2005 [note 185], ch. VI.
} 
dans le choix des solutions possibles a pour conséquence d'augmenter la proportion en faveur de la solution médiane ${ }^{193}$.

Ces conclusions sont transposables dans le domaine de la soft law. N'est-ce pas sur ce type de réaction ${ }^{194}$ que parie la nouvelle carte de donneur d'organe en Suisse proposant dorénavant trois possibilités ${ }^{195}$ : l'autorisation de tout prélèvement d'organe, la non-autorisation et, à titre de solution intermédiaire, le prélèvement sélectif (cases à cocher) de divers organes tels que le cœur, les poumons, le foie, les reins, l'intestin grêle, le pancréas, la cornée, la peau ainsi que d'autres tissus et cellules? Une étude resterait à faire, mais on peut raisonnablement poser l'hypothèse que la présence d'options intermédiaires sera évaluée plus favorablement. Ce type de mesure ne pose pas de problème particulier du point de vue des principes de l'Etat de droit, étant une expression du principe de proportionnalité (art. 5 al. 2 Cst.).

\section{CONCLUSION}

Les Etats ont constaté depuis longtemps l'inefficacité des instruments juridiques classiques dans le pilotage des politiques publiques. Le soft power $^{196}$ est redécouvert, thématisé et utilisé avec une efficacité parfois étonnante, en particulier en synergie avec les moyens juridiques traditionnels. L'impact de la soft law est d'autant plus fort que cette dernière, dans ses stratégies persuasives, sait habilement faire appel aux émotions, voire à des techniques de manipulation.

La question est de savoir jusqu'à quel point les autorités peuvent faire vibrer les cordes de l'émotion pour faire progresser le taux de respect des instruments de soft law, sachant que ces derniers, dans un Etat de droit, n'ont pas la même légitimité démocratique que les moyens législatifs et réglementaires. L'Etat ne peut ainsi pas employer n'importe quelle méthode de manipulation pour développer la médecine de transplantation; mais pourrait-il, comme on le voit dans les campagnes de communication en sécurité routière, tabler sur une heuristique de la simulation, en montrant un scénario catastrophe hypothétique conduisant à un accident, afin de rendre l'accident plus probable aux yeux des destinataires? Fournir un tel scénario à un individu rend en effet, selon les recherches en psychologie sociale, l'événement plus vraisemblable à ses yeux ${ }^{197}$.

La mise en évidence des effets produits par la soft law à l'aune des émotions suscitées montre que des actes non juridiquement obligatoires peuvent être contraignants. L'absence d'effets juridiques au sens classique ne saurait donc leur permettre de se soustraire à l'adoption d'un régime de légitimation et de contrôle adapté que les règles de hard law connaissent depuis longtemps dans les Etats de droit. Il importerait en conséquence d'assujettir les actes de soft law - toutes

${ }^{193}$ Schweizer 2005 [note 185], ch. VI, lit. C.

${ }^{194}$ On peut également se référer à la technique du «un peu-c'est-mieux-que-rien» (Joule/ Beauvois 2002 [note 153], p. 162).

195 Voir www.swisstransplant.ch

${ }^{196}$ Nye, Joseph, Bound to Lead: The Changing Nature of American Power, New York 1990.

${ }^{197}$ Joule/Beauvois 2006 [note 152], p. 202. 
proportions gardées et en fonction de leurs impacts, émotionnels en particulier aux principes matériels de l'Etat de droit ${ }^{198}$ tels que la légalité, l'action dans l'intérêt public, le respect de la bonne foi et de la proportionnalité (art. 5 Cst.), l'exigence d'un traitement égal et non discriminatoire (art. 8 Cst.), la prohibition de l'action arbitraire (art. 9 Cst.), ainsi qu'à des règles formelles telles que la définition de garanties procédurales spécialement adaptées comme le recours à la médiation ou, dans certains cas, aux voies juridictionnelles ${ }^{199}$. Des mécanismes de consultation et de participation du public devraient également être développés dans le processus d'adoption des règles de soft law, à l'instar de forums-citoyens et de conférences de consensus, de sorte à asseoir une véritable «démocratie administrative ${ }^{200}$. Une légitimité accrue de tels actes en renforcerait par ailleurs l'efficacité comme les travaux en psychologie sociale l'ont montré201.

Cette conclusion ne délégitime en aucune mesure le recours de la soft law aux émotions, voire à certaines techniques non problématiques et bien ciblées de manipulation; elle demande simplement un encadrement juridique adapté aux spécificités des actes étatiques non obligatoires afin de ne pas contourner les principes de l'Etat de droit. L'efficacité même de la soft law serait sinon tout bonnement remise en question, à considérer l'importance et l'étendue des effets affectifs qu'elle est à même d'éveiller.

Faculté de droit, Université de Genève

\footnotetext{
${ }^{198}$ En détail sur cette proposition, voir Flückiger 2004 [note 11], p. 262ss.

${ }^{199}$ Flückiger 2004 [note 11], p. 270ss.

${ }^{200}$ Flückiger 2004 [note 11], p. 275ss

${ }^{201}$ Voir les travaux de Tyler 2006 [note 51] à propos de l'obéissance à la loi, mais dont les conclusions peuvent à notre avis être transposées au droit souple.
} 